\title{
An Automated Antenna Measurement System Utilizing Wi-Fi Hardware
}

\author{
BY \\ KEITH ROBERT MARTIN \\ B.S., Northwestern University, 2007
}

\begin{abstract}
THESIS
Submitted as partial fulfillment of the requirements

for the degree of Master of Science in Electrical and Computer Engineering in the Graduate College of the University of Illinois at Chicago, 2011
\end{abstract}

Chicago, Illinois

Defense Committee:

Danilo Erricolo, Chair and Advisor

Sharad R. Laxpati

Piergiorgio L.E. Uslenghi 


\section{ACKNOWLEDGMENTS}

I would like to express my sincere gratitude to my advisor, Professor Danilo Erricolo. I would also like to thank my collaborator in this project, Vittorio Picco. I would not have been able to complete this project without the guidance and assistance of Professor Sharad Laxpati, Professor Konrad Kaczmarski, Tadahiro Negishi, and Haijiang Ma. Finally, I would like to thank the IEEE Antennas and Propagation Society for their support of this project and for hosting the Student Design Competition.

KRM 


\section{TABLE OF CONTENTS}

CHAPTER

$\underline{\text { PAGE }}$

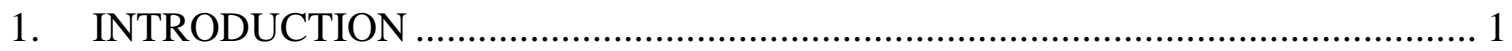

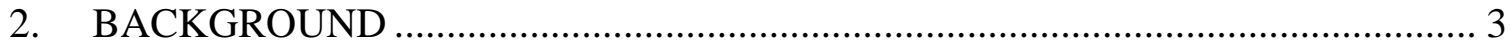

2.1. The Necessity for Antenna Pattern Measurements ........................................... 3

2.2. Theory of Antenna Measurements.................................................................... 3

2.3. Types of Far-Field Antenna Ranges .............................................................. 4

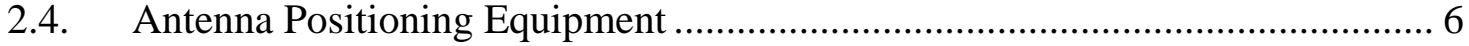

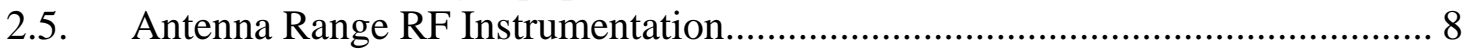

2.6. Antenna Gain Measurement Methods ............................................................. 9

2.6.1. The Absolute-Gain Measurement Methods ............................................... 9

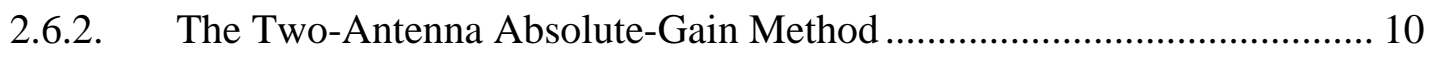

2.6.3. The Three-Antenna Absolute-Gain Method ............................................ 10

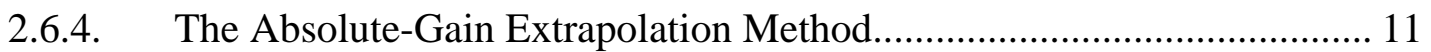

2.6.5. The Gain-Transfer Measurement Methods .............................................. 12

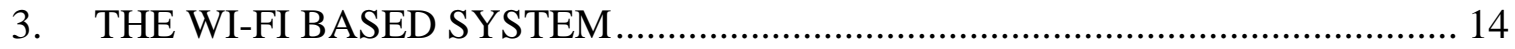

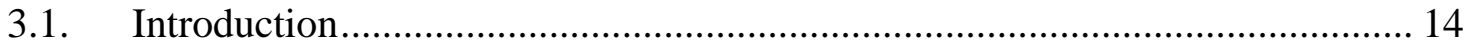

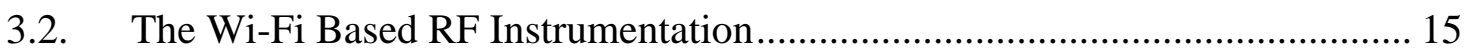

3.3. The Antenna Positioner................................................................................ 20

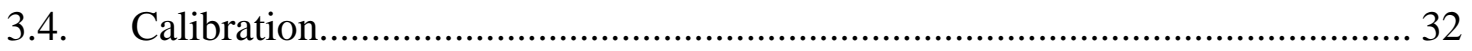

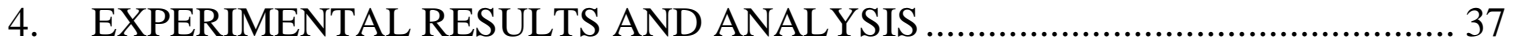

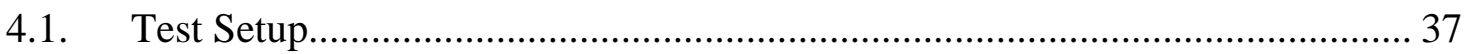

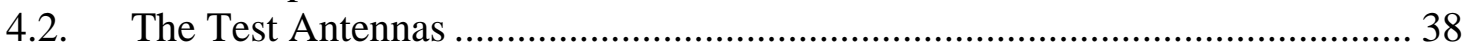

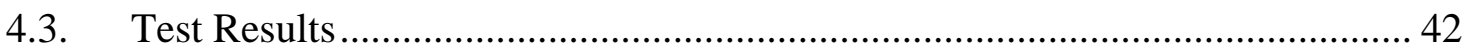

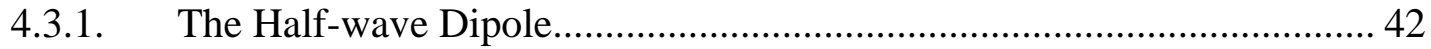

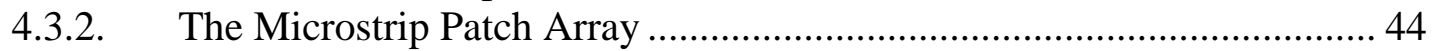

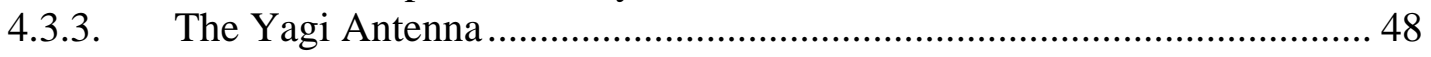

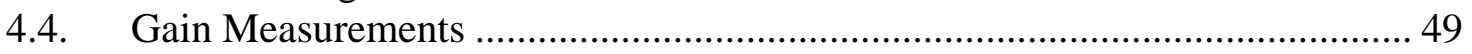

5. FUTURE WORK AND CONCLUSION ............................................................ 55

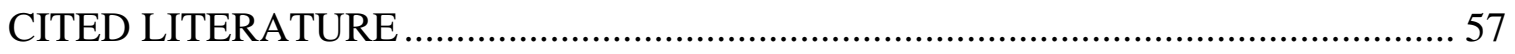

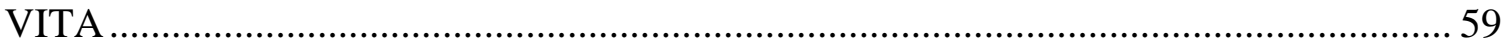




\section{LIST OF TABLES}

TABLE

$\underline{\text { PAGE }}$

1. ABSOLUTE GAIN MEASUREMENT, YAGI ANTENNA .................................. 52

2. ABSOLUTE GAIN MEASUREMENT, MICROSTRIP ARRAY ANTENNA........ 53 


\section{LIST OF FIGURES}

FIGURE

$\underline{\text { PAGE }}$

1. An elevation over azimuth positioner. Each scan can be a great-circle (a) or conical section (b)

2. Normalized radiation pattern of the dipole antenna supplied with the router, measured to test the viability of a Wi-Fi based antenna measurement system. The characteristic pattern of a dipole is visible

3. The initial design of the positioner created using Google SketchUp....

4. The turntable bearing attached to the plywood base. A disk is attached to the top of the bearing. Holes drilled through the base permit access to the screws used to retain the disk

5. The yaw axis disk mounted on top of the turntable bearing. The support for the yaw axis motor has also been attached to the plywood base

6. The completed roll axis assembly mounted on top of the column. The additional hole in the bearing block provides a path to route a coaxial cable

7. The stepper motors and their belt drives. The yaw axis motor is suspended from the brace at the right. The roll axis motor is fixed to the base of the column using angle iron brackets.

8. The roll axis motor and its custom fabricated mounting bracket, attached to the base of the column. Note the rubberized drive wheel fitted to the shaft of the motor

9. The LabVIEW GUI. Users can manually rotate the positioner, take RSSI measurements, set the port address and positioner gear ratios, and perform automated scans and view and export results

10. Received power measurements produced using a spectrum analyzer and the Wi-Fi router. Transmitted power is reported by the transmitting router.

11. Difference between the RSSI value returned by the router and the spectrum analyzer measurement as a function of the RSSI obtained from the router.

12. The test setup used to evaluate the system, showing the positioner, routers, and controlling computer located in an outdoor environment. 


\section{LIST OF FIGURES (continued)}

FIGURE

PAGE

13. The $3 \mathrm{GHz}$ half wave dipole. The directions used in the scan are indicated 40

14. The Wi-Fi Yagi antenna. The directions used in the scan are indicated................... 41

15. The microstrip patch array antenna. The directions used in the scan are indicated... 41

16. Normalized $\theta$ plane pattern of a dipole obtained using the Wi-Fi system. The simulated behavior of this dipole is superimposed over the measured results

17. Normalized $\varphi$ plane pattern of a dipole obtained using the Wi-Fi system. The simulated behavior of this dipole is superimposed over the measured results

18. Normalized $\theta$ plane pattern of a microstrip array obtained using the Wi-Fi system.

The simulated behavior of this antenna is superimposed over the measured results.. 45

19. Normalized $\varphi$ plane pattern of a microstrip array obtained using the Wi-Fi system.

The simulated behavior of this antenna is superimposed over the measured results.. 46

20. Normalized $\theta$ plane pattern of a microstrip array obtained using the Wi-Fi system scanning the roll axis in the clockwise direction.

21. Normalized $\theta$ plane pattern of a microstrip array obtained using the Wi-Fi system scanning the roll axis in the counter-clockwise direction.

22. Normalized $\theta$ plane pattern of a Yagi antenna obtained using the Wi-Fi system. The simulated behavior of this antenna is superimposed over the measured results.. 49 


\section{LIST OF ABBREVIATIONS}

\begin{tabular}{ll} 
AP-S & Antennas and Propagation Society \\
AUT & Antenna Under Test \\
CNC & Computer Numeric Control \\
GUI & Graphical User Interface \\
IEEE & Institute of Electrical and Electronics Engineers \\
JTAG & Joint Test Action Group \\
MA & Measurement Antenna \\
PCB & Printed Circuit Board \\
RF & Radio Frequency \\
RP-TNC & Reverse-Polarity Threaded Neill-Concelman \\
RSSI & Received Signal Strength Indicator \\
SGA & Service Set IDentifier \\
SMA & SubMiniature version A \\
SSH & Secure SHell \\
\hline
\end{tabular}




\section{SUMMARY}

A novel method of measuring antenna radiation patterns and gains is presented in response to the 2011 IEEE Antenna and Propagation Society Student Design Challenge. This challenge requires entrants to produce a system to measure radiation patterns and gain that can be reproduced for less than $\$ 1500$.

To determine the requirements for this system, traditional antenna measurement instruments and techniques are explored. A summary of antenna measurement principles is provided, and is used as a guide for the design of this system.

IEEE 802.11b Wi-Fi routers are used to generate a test signal and measure the power received by a test antenna. Rather than attempt to replicate the RF instrumentation used in traditional antenna measurement systems, this system utilizes an inexpensive and readily available consumer product. A calibration procedure and dithering are employed to reduce error to less than $0.5 \mathrm{~dB}$. Gain transfer methods are used to produce absolute gain measurements. This use of a consumer product to replace an instrument is one of the most novel aspects of this system and a key contributor to its low cost and simple implementation.

An antenna positioner is designed and fabricated using readily available materials and rudimentary techniques. This positioner allows precise control of antenna rotation in both principal planes. Stepper motors are used to drive each axis of the positioner, and are controlled through a PC parallel port interface. The stepper motors and drivers are obtained from a kit intended for hobbyist CNC milling machines. This kit provides an easy and inexpensive alternative to designing and building a custom stepper motor drive. 


\section{SUMMARY (continued)}

Antenna radiation patterns and gains are measured for several inexpensive antennas. These results are compared to analytical results and simulations produced using FEKO. These results demonstrate the viability of this system. Further enhancements are also presented.

The cost to reproduce the system is $\$ 924.46$, well under the $\$ 1500$ limit. This system was selected as a finalist by the IEEE AP-S committee, and was demonstrated at the 2011 AP-S Symposium in Spokane, Washington. The system was ultimately awarded $3^{\text {rd }}$ prize. 


\section{INTRODUCTION}

This work describes an antenna measurement system that seeks to replicate the

performance of a commercial system at a significantly reduced price. This system is designed using the same principles that guide the design of commercial antenna ranges, but utilizes repurposed consumer electronic equipment, widely available materials, basic construction techniques, and common student licensed software.

The motivation for this work is the 2011 Institute of Electrical and Electronics Engineers Antennas and Propagation Society (IEEE AP-S) Student Design Challenge. This competition challenges entrants to design a system to measure antenna patterns that can be reproduced for under $\$ 1500$. The system need not operate in an anechoic chamber, but should be designed to deliver gain measurements accurate to $\pm 0.5 \mathrm{~dB}$ in an anechoic environment. It also needs to operate at $2.4 \mathrm{GHz}$. Free or common student licensed software and a personal computer may be used, and the cost of the personal computer need not be included in the $\$ 1500$ limit. Finalists selected by the IEEE AP-S will be required to demonstrate their systems at the 2011 IEEE AP-S Symposium in Spokane, Washington. The system will need to be disassembled, transported to the conference, and reassembled for the demonstration. It must also be compact enough to be displayed on a tabletop. Based on a preliminary proposal, the IEEE AP-S provided the funds to develop and construct this system. The resulting system was selected to be one of the three finalists, and was presented to judges and conference attendees at the 
IEEE AP-S Symposium. The system was ultimately awarded $3^{\text {rd }}$ prize during the symposium awards ceremony.

This work examines the theories and techniques used in traditional antenna ranges, with emphasis on the equipment required to perform antenna pattern measurements. The system entered into the 2011 IEEE AP-S Student Design Challenge is described in detail. Experimental results are presented and compared to simulation and analytical results for several test antennas. Further enhancements to the system will also be proposed. 


\section{BACKGROUND}

\subsection{The Necessity for Antenna Pattern Measurements}

Pattern measurements are essential to determining the performance of antennas and assessing compliance with simulation results and design criteria. Finding an analytical solution for all but the simplest antenna geometries can be prohibitively difficult. New computational techniques have allowed many antenna types to be examined with simulators, but obtaining simulation results can require long computation times. Simulation results may also need to be validated empirically to ensure that the desired pattern is obtained [1]. This can only be accomplished through pattern measurement. Pattern measurements allow the performance of an antenna to be conclusively determined when analytical or simulation results are questionable or difficult to obtain. These measurements are always considered to be more definite than any analytical or simulation results.

\subsection{Theory of Antenna Measurements}

A pattern measurement is obtained by measuring the power transmitted or received by an antenna over a constant radius sphere [2]. The antenna to be measured is referred to as the Antenna Under Test (AUT). This antenna may transmit a signal to an additional antenna, known as the Measurement Antenna (MA), or receive a signal transmitted by the MA. According to the reciprocity principle, operating the AUT as 
either the transmitter or receiver will yield the correct result. However, for practical reasons the AUT is typically operated as a receiver. This allows the transmitter to be fixed, preventing any variation in transmitter performance due to mechanical vibrations. The receiver is typically smaller and lighter than the transmitter, making it easier to move and less susceptible to the effects of vibration [3]. Rotating the AUT allows the received power at a fixed radius to be measured as a function of $\theta$ and $\varphi$, the angular components of a spherical coordinate system. Antenna patterns are three-dimensional, and can be observed by sampling the entire three-dimensional space around the antenna. The number of points sampled is determined based on the antenna's design requirements and the amount of time and funding available. Generating three-dimensional pattern measurements requires significantly more samples than a principal plane pattern measurement. The principal planes of a linearly polarized antenna are the planes containing the electric and magnetic field vectors. Patterns are often measured in these planes only, reducing the number of samples required. When performing a principal plane pattern measurement, the antenna's principal planes should coincide with the coordinate planes. This simplifies the analysis of results and the identification of measurement anomalies [4].

\subsection{Types of Far-Field Antenna Ranges}

Antenna pattern measurements are typically conducted in dedicated antenna ranges. An antenna range contains fixtures to mount antennas on, an RF system to feed one antenna and measure the received power at the other antenna, and a system to record 
the measured results. These components are often computer controlled, allowing the measurement procedure to be automated. Pattern measurements are usually performed to obtain the AUT's far field radiation pattern, so the AUT and MA must be located in each other's far field region. This is the region where the incident wave can be approximated as a plane wave. The distance between the two antennas necessary to obtain this condition is dependent on the size of the antenna and the operating wavelength. The distance to the far field region is expressed by the following relation [5]:

$$
r \geq \frac{2 D^{2}}{\lambda}
$$

where $D$ is the largest dimension of the antenna aperture, $r$ is the distance between the antenna and the observation point, and $\lambda$ is the operating wavelength. The antenna's size is relevant because it determines the distance at which the vectors between two points on the antenna and the observation point can be approximated as parallel [6].

In the far field region, the spherical incident wavefront has a large enough radius that it behaves as a plane wave over the aperture of the antenna. The above relation shows that this occurs when the phase difference over the antenna aperture is less than $\lambda / 16$, or $22.5^{\circ}[7]$.

When operating at short wavelengths and with compact structures, antenna ranges can be constructed in enclosed anechoic chambers. These chambers use their geometry and RF absorbing material to reduce the effect of reflections. RF absorbing material becomes less effective as the incidence angle moves away from the normal vector. The geometry of the room is designed to limit incidence at large angles to the normal and to 
reduce the effects of these reflections [8]. For long wavelengths or confined spaces, special techniques are required, such as the use of reflectors to generate plane waves in a smaller physical space.

Outdoor ranges are used when building an indoor range is impractical. Care must be taken to minimize the effect of reflections and interference in these ranges. Several methods are used to accomplish this. Time gating can be used to exclude signals based on their multipath delay. This method requires sufficient bandwidth in the measurement system to detect delayed components of the test signal. This is especially important when reflections occur over small physical distances, resulting in short delays between multipath components. Illuminating the AUT with a directive MA also reduces the amount of off-axis reflection from the environment. To reject interfering signals, the transmitted signal may be modulated [9]. The signal received by the AUT is demodulated by the receiver, allowing only the power in the desired signal to be measured.

\subsection{Antenna Positioning Equipment}

Antenna ranges can be equipped with a variety of fixtures to rotate the AUT through the desired observation angles. Two orthogonal axes are required to perform scans in both the $\theta$ and $\varphi$ axes. These can be obtained by mounting the AUT on a column that can rotate in one axis and that supports the other axis. Two configurations are possible-azimuth over elevation and elevation over azimuth. The former places the $\varphi$ axis over the $\theta$ axis, resulting in a sphere with its z-axis coincident with the azimuth 
rotation axis. The latter configuration results in a sphere with its z-axis coincident with the elevation rotation axis [10]. The scans possible with an elevation over azimuth positioner are shown in Figure 1 [11]. Great circle scans are performed by scanning the entire azimuth axis for a given value of the elevation axis. Conical section scans are performed by scanning the entire elevation axis for given values of the azimuth. The conical section scan is preferred because it allows a spherical coordinate system to be used without difficult coordinate transformations. The terms yaw and roll are also used in place of azimuth and elevation, respectively, to describe these axes.

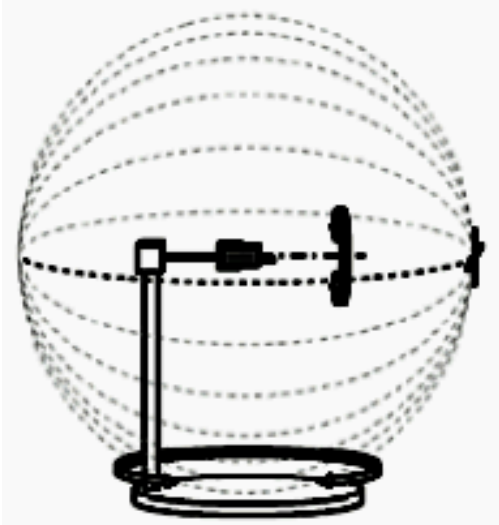

(a)

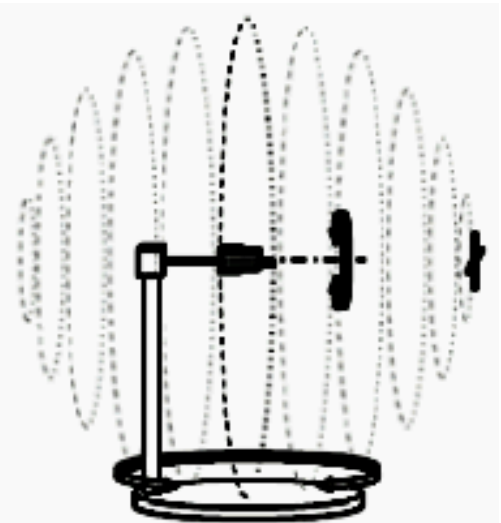

(1)

Figure 1: An elevation over azimuth positioner. Each scan can be a great-circle (a) or conical section (b)

To sample orthogonal linear polarizations, the MA can also be mounted on a positioner. This positioner rotates the MA into the desired polarizations. Alternatively, an MA that can switch polarizations electrically is used.

Antenna positioners may introduce errors through misalignment of their axes. This can include misalignment of the positioner axes with the principal planes of the 
AUT, axes that are not perfectly orthogonal, eccentricity in the axes, and errors in axis positioning. Axis positioning errors on commercial positioners are typically smaller than $0.05^{\circ}$. To prevent the positioner from altering the pattern of the AUT or distorting the measurement through electromagnetic scattering, it may support the antenna on a nonmetallic boom or a Styrofoam column [12].

\subsection{Antenna Range RF Instrumentation}

An antenna range also requires RF instrumentation. This consists of a signal generator to drive the transmitter, and a receiver to measure the received signal power. Commercial antenna ranges are designed to operate at a range of frequencies, which increases the complexity of the RF instrumentation. The transmitter needs to accurately control and maintain the desired frequency, power, and modulation. The receiver must demodulate and accurately measure the incident signal. Designing a receiver with high precision and dynamic range can be difficult and expensive [13]. If time gating is being used to exclude multipath components, this feature must also be implemented in the receiver. Recording equipment allows the position and pattern measurements to be plotted and saved for later analysis.

Modern antenna ranges are computer automated, allowing central control of the RF instrumentation and positioner. The RF instrumentation often includes a network analyzer, and the computer interfaces with the network analyzer to record the pattern measurements [14]. 


\subsection{Antenna Gain Measurement Methods}

Antenna gain measurements are essential to determining wireless system performance. Two basic methods are used to obtain gain measurements-the absolutegain method, and the gain-transfer method. The absolute-gain method does not require the gain of any of the antennas used to be known beforehand. Therefore, it can be used when a Standard Gain Antenna (SGA) is unavailable, or to produce a SGA. This SGA can then be used to make gain measurements using the gain-transfer method.

\subsubsection{The Absolute-Gain Measurement Methods}

Absolute-gain measurements can be produced using several techniques. These techniques all rely on the Friis transmission formula, shown below [15].

$$
\frac{P_{r}}{P_{t}}=\left(\frac{\lambda}{4 \pi R}\right)^{2} G_{0 t} G_{0 r}
$$

In decibel form, the Friis formula can be expressed as

$$
G_{0 t}+G_{0 r}=20 \log _{10} \frac{4 \pi R}{\lambda}+10 \log _{10} \frac{P_{r}}{P_{t}},
$$

where $G_{0 t}$ and $G_{0 r}$ are the gain of the transmitting and receiving antennas and $P_{t}$ and $P_{r}$ are the transmitted and received powers, respectively. The distance between the two antennas is $R$, and the operating wavelength is $\lambda$.

To minimize errors when using absolute-gain methods, the antennas must be mounted so that the far-field condition is satisfied and both antennas are aligned in the maxima of their patterns. The antennas also need to be impedance and polarization matched. If the antennas are not impedance and polarization matched, their complex 
impedance and polarization must be known so that correction can be applied. This requires additional instrumentation. Complex impedance can be measured using a network analyzer, while polarization measurements require rotating the antenna to sample multiple polarizations or the use of multiple polarization antennas. Impedance mismatches may also be remedied using matching networks [16]. The Friis formula also assumes that no energy is received through reflection from the environment. Care must be taken to prevent reflections from affecting the result. Reflections from the antennas themselves are especially difficult to remove, and are typically evinced by a periodic variation in gain as the separation between the antennas is varied [17].

\subsubsection{The Two-Antenna Absolute-Gain Method}

If the two antennas are identical, the Friis formula can be further simplified.

$$
\left(G_{0 t}\right)_{d B}=\left(G_{0 r}\right)_{d B}=\frac{1}{2}\left[20 \log _{10} \frac{4 \pi R}{\lambda}+10 \log _{10} \frac{P_{r}}{P_{t}}\right]
$$

This allows the gain of the antennas to be found using the measured values of $R, \lambda$, and $\frac{P_{r}}{P_{t}}$

\subsubsection{The Three-Antenna Absolute-Gain Method}

The previous method requires the use of two identical antennas. If two identical antennas are not available, an absolute-gain method measurement can also be performed using 
three antennas. Three measurements must be made, and three equations written to represent three combinations of the antennas. The following equations are used for three antennas referred to as a, b, and c.

$$
\begin{aligned}
& G_{a}+G_{b}=20 \log _{10} \frac{4 \pi R}{\lambda}+10 \log _{10} \frac{P_{r b}}{P_{t a}} \\
& G_{a}+G_{c}=20 \log _{10} \frac{4 \pi R}{\lambda}+10 \log _{10} \frac{P_{r c}}{P_{t a}} \\
& G_{b}+G_{c}=20 \log _{10} \frac{4 \pi R}{\lambda}+10 \log _{10} \frac{P_{r c}}{P_{t b}}
\end{aligned}
$$

The gains of all three antennas can be determined by solving these equations with the measured values of $R, \lambda$, and the measured power ratios in each combination.

\subsubsection{The Absolute-Gain Extrapolation Method}

Due to the possibility of errors when using the three-antenna method, an extrapolation technique can be applied. This method can provide the gain and polarization of all three antennas if all are nominally linearly polarized. If one antenna is circularly polarized, only this antenna can be fully characterized. If more than one antenna is circularly polarized, this method cannot be used.

The extrapolation technique requires that the received power be measured while the separation between the antennas is varied. Measuring received power is sufficient for gain measurements; to determine polarization the phase must be measured as well. The effects of multipath interference as the antennas are moved are averaged out 
mathematically. A function that describes the received power as a function of separation can then be defined. This function then allows the received power in the far-field to be extrapolated.

Antenna ranges capable of performing extrapolation measurements are rare and require unique equipment. The antennas need to be mounted so that they remain boresighted as the separation between them is varied. Measuring antennas with large gains requires greater separation between the antennas and taller mounting structures. These large, movable structures are difficult and expensive to build. Therefore, extrapolation ranges are often available only to government institutions such as the National Bureau of Standards [18].

\subsubsection{The Gain-Transfer Measurement Methods}

The gain-transfer method is more commonly used than the absolute-gain methods. This technique relies on the use of Standard Gain Antennas (SGAs) with known and stable gain values. The relative gain of an unknown antenna is compared to the known gain of the SGA, resulting in an absolute gain measurement. This method requires a measurement to be performed with the AUT, and a measurement with the SGA. All other experimental conditions must remain identical when the two measurements are taken. In the first measurement, the amount of power received with the AUT is measured. Then the amount of power received with the SGA under the same conditions is measured. The difference in gain between the SGA and the AUT is determined by the 
difference in received power in each experiment. In logarithmic decibel units, this can be expressed as

$$
G_{A U T}=G_{S G A}+10 \log _{10} \frac{P_{A U T}}{P_{S G A}}
$$

This method suffers less from multipath effects, especially if the SGA is similar to the AUT. As in other methods, if the antennas are polarization or impedance mismatched the appropriate corrections must be determined and applied. To minimize disruptions between the two experiments, the antennas can be mounted back-to-back on a turntable. Rotating the turntable would move one antenna into the exact position used for the previous measurement. Additional measurements or the use of an SGA with the same polarization as the AUT allow the gain of non-linearly polarized antennas to be measured. 


\section{THE WI-FI BASED SYSTEM}

\subsection{Introduction}

To address the shortcomings of traditional antenna ranges, an alternative system is proposed. The expense of installing a dedicated antenna range and its required positioners, RF instrumentation, and gain standards prevents many organizations from performing antenna measurements. While commercial antenna ranges are required for critical applications and the certification of commercial gain standards, other users may benefit from a less expensive system that requires only minor compromises in versatility and accuracy.

This alternative system employs the same theories used in the design of commercial antenna ranges, but seeks to replicate its components with less expensive and more widely available materials. Instead of an enclosed anechoic chamber, measurements are performed outdoors. Making the system easily portable allows users to move it to suitable outdoor locations when a measurement is needed. The RF instrumentation is replaced with Wi-Fi routers. This substitution alone eliminates the enormous expense of

purchasing an instrument, and the difficulty of designing a custom equivalent. A positioner is constructed out of readily available materials using basic techniques. Finally, a common personal computer and student licensed software allow automated measurements and visualization of results. Each of these subsystems is described in further detail below. 


\subsection{The Wi-Fi Based RF Instrumentation}

Rather than attempt to replicate the instrumentation used in commercial systems, this work demonstrates that an existing consumer product can be used to measure antenna patterns. Wi-Fi routers provide a number of features that make them especially suitable this application. A router serving as an access point will broadcast a specified Service Set Identifier (SSID). A second router measures the power of the received RF signal with the same SSID. Information contained in the preamble of a Wi-Fi frame allows the receiving router to distinguish between the signals in the channel and to compute only the power in the desired signal. This is analogous to the use of modulated signals in outdoor antenna ranges to determine which incident signal is desired. This measurement is available to the user as the Received Signal Strength Indicator (RSSI), and is intended to help users assess the quality of a wireless connection. RSSI is a dimensionless parameter and is measured relative to an arbitrary maximum value, RSSI_Max [19]. The value of RSSI_Max and quantization of RSSI are not specified in the IEEE 802.11 standards, and may vary among vendors. Linksys brand WRT54G routers use an RSSI_Max of 100, and provide RSSI as an integer between -100 and 0 [20]. This is the finest quantization available in commercial routers, resulting in better sensitivity to small RSSI variations and improved accuracy in pattern measurements. To produce an absolute gain measurement from RSSI requires a calibration procedure, which will be described below. This capability to produce and measure an RF signal makes Wi-Fi routers a viable RF subsystem for an antenna measurement system. 
The IEEE 802.11b standard describes a system of $22 \mathrm{MHz}$ channels, with the first channel centered on $2.412 \mathrm{GHz}$. The channel system allows users to select a channel that minimizes interference from other nearby Wi-Fi users. Because of the requirement that the system operate at $2.4 \mathrm{GHz}$, channel 1 -the lowest frequency channel—is used. This satisfies the requirement that the system operate at 2.4 GHz. Other Wi-Fi standards, such as IEEE $802.11 \mathrm{a} / \mathrm{n}$, allow operation at $5 \mathrm{GHz}$. The use of routers designed for these standards allows this technique to be used to measure antenna patterns at an alternate frequency.

To confirm that routers are suitable for use in antenna measurement, a test was conducting in the anechoic chamber at the Andrew Electromagnetics Laboratory at UIC. The routers are supplied with dipole antennas. If a pattern measurement using one of these antennas as the AUT revealed the toroidal pattern of a dipole, the method was sound. The transmitting router was connected to the standard anechoic chamber MA, an S-band rectangular waveguide antenna. The receiving router and its manufacturer supplied antenna were attached to a two-axis positioner in the anechoic chamber. A principal plane measurement was taken by manually rotating the positioner and recording the RSSI in each position. The resulting graph, shown in Figure 2, shows the expected pattern of a dipole, albeit with some explainable anomalies. The results proved to be highly repeatable, with the same anomalies occurring in each measurement. This means that they are likely the result of a physical phenomenon, and not a random process. 


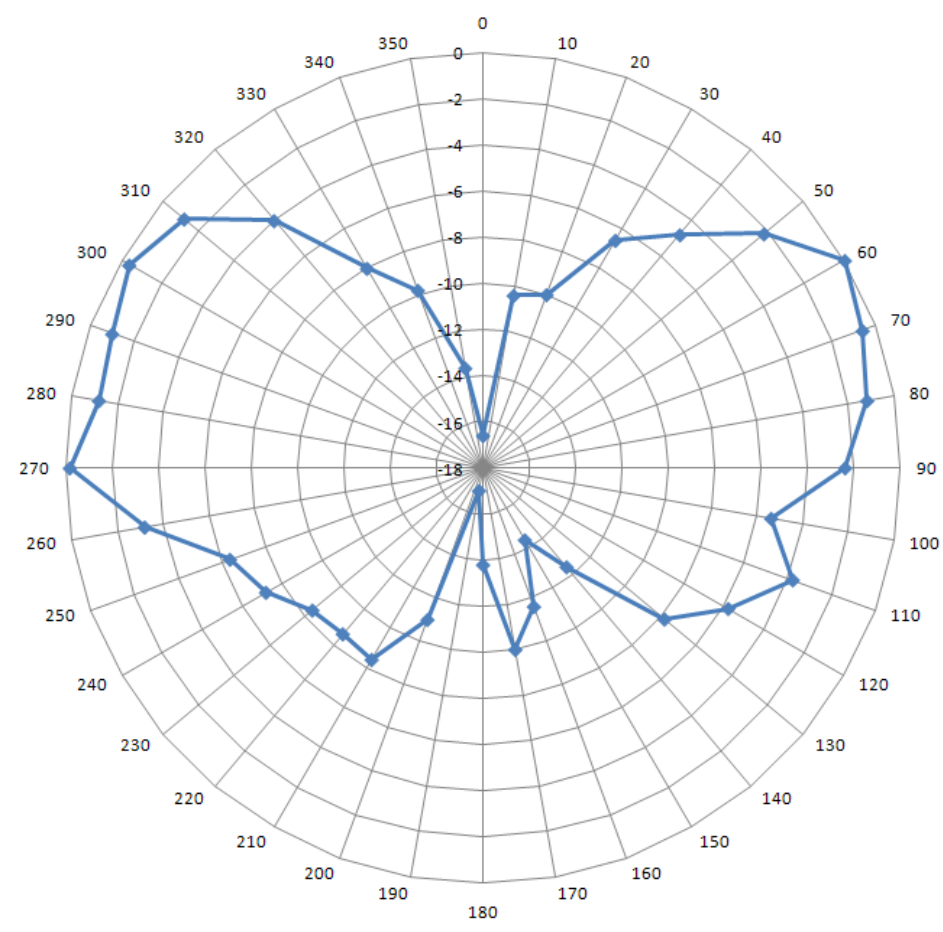

Figure 2: Normalized radiation pattern of the dipole antenna supplied with the router, measured to test the viability of a Wi-Fi based antenna measurement system. The characteristic pattern of a dipole is visible.

The asymmetrical nature of the dipole pattern is believed to be caused by scattering from the metal structure of the positioner and the body of the router. The notches in the pattern at $150^{\circ}$ and $100^{\circ}$ coincide with the position of the metal boom used to support the router. The asymmetry between the left and right halves of the pattern is the result of the antennas being obscured by the body of the router. These anomalies were present in all repetitions of the test. The finished system mounts the AUT on a dielectric boom, and locates the router off of the positioner, eliminating these problems.

Wi-Fi routers provide several additional advantages beyond their ability to generate and measure RF signals. The $2.4 \mathrm{GHz}$ band is unlicensed and widely used by consumer devices, such as Wi-Fi and Bluetooth (IEEE 802.15) devices, cordless phones, 
and microwave ovens. When working outside the shielded environment of an anechoic chamber, these interfering signals could distort pattern measurements. Therefore, to accurately measure antenna patterns, the receiver must exclude these undesired signals. Because RSSI is computed using information contained in the preamble of a Wi-Fi frame, RSSI provides better interference robustness than a simple measurement of received power in the channel. This ensures that only the desired signal is measured, improving the accuracy of the result.

Wi-Fi routers also provide an interface to communicate with a computer, allowing the RSSI measurement to be retrieved automatically. National Instruments LabVIEW software is used to integrate the functions of retrieving RSSI measurements, controlling positioner movements, and recording measurement results. Student licenses for LabVIEW are inexpensive, and the competition rules allow the use of common, student licensed software. Communication with the controlling computer using LabVIEW is facilitated by a Secure SHell (SSH) interface to the router. Open source firmware has been developed for many routers, allowing increased control of router parameters and often incorporating additional features such as SSH interfaces. Linksys WRT54G v.5 and v.6 routers are used in this project, as they support the micro-plus version of dd-wrt open source router firmware. The micro-plus version of this firmware incorporates an $\mathrm{SSH}$ interface that is not present in the micro version. The amount of available flash memory in the router limits how many additional features can be incorporated into the firmware. When using WRT54G v.5 and v.6 routers, the router's bootloader must be compressed prior to flashing with the micro-plus firmware. This creates additional space 
to accommodate the SSH feature. This process was overlooked initially, requiring a reflash of the routers using a Joint Test Action Group (JTAG) interface to restore functionality. To perform a reflash using JTAG, a parallel port connector must be assembled and soldered to a JTAG header on the router. This procedure restores the router to its original configuration. The Linksys WRT54G series of routers has a large user community, which provides extensive support and documentation for these routers. The dd-wrt firmware is maintained by the community at dd-wrt.org, and the source files and detailed procedures used to install the firmware are described there.

The Linksys WRT54G has detachable antennas, allowing the user to attach the desired MA and AUT. The router uses RP-TNC type connectors, an unusual type of connector intended to deter end users from installing incompatible antennas. Adapters are needed to connect standard coaxial cables. These can be purchased inexpensively from a number of electronics retailers. Which of the router's two antennas to use as the transmit and receive antenna can be specified using the dd-wrt firmware, and the MA and AUT are attached accordingly.

Although the dd-wrt firmware allows enhanced control of the routers, it does not allow significant modifications to their behavior. This makes implementing complex functions like time gating impossible. Therefore, alternate methods of reducing the effect of reflections are used. In this application the MA functions as the transmitting antenna, and the AUT as the receiver. When working outside of an anechoic environment, a directive MA reduces the amount of power received through off-axis reflections from the environment. Wi-Fi's large user base makes a wide variety of directional $2.4 \mathrm{GHz}$ 
antennas available. These antennas are often implemented on printed circuit boards (PCBs) for low-cost, high-volume manufacture. Microstrip patch arrays, log-periodic dipole arrays, and Yagi antennas are all available at electronics retailers. The patch array antenna has the highest nominal gain of the PCB antennas readily available from online retailers, $11.5 \mathrm{dBi}$. It is also linearly polarized, as are all Wi-Fi antennas.

The routers, required adapters, and Wi-Fi antennas can be purchased for less than $\$ 200$. The low price of the RF subsystem is a key factor in allowing this system to be recreated for well under the goal of $\$ 1500$. Using Wi-Fi hardware eliminates the time and expense required to develop, manufacture, and assemble custom RF hardware. By allowing the use of RSSI instead of a simple power measurement, Wi-Fi also has the potential to produce superior results in the noisy environment outside an anechoic chamber. This use of an inexpensive, readily available technology for an unintended purpose is one of the most novel aspects of this system.

\subsection{The Antenna Positioner}

The antenna positioner is similar in principle to commercial offerings, but is designed to be implemented at low cost and without sophisticated manufacturing equipment. It is constructed with its roll axis over its yaw axis. Movement in the yaw axis corresponds to movement in the $\theta$ direction, while movement in the roll axis corresponds to movement in the $\varphi$ direction. The coordinate sphere around the antenna can be imagined as having its poles in the $y$-axis of a right-handed coordinate system, with its equator in the $x z$-plane. 
To simplify the procurement and fabrication of the positioner's components, wood is used as its primary construction material. Wood is inexpensive and a large selection of wood products can be purchased at home improvement stores. Wood structures can be fabricated without sophisticated techniques and equipment. Finally, wood has benign electrical characteristics, and will not cause excessive electromagnetic scattering or reflection.

The initial design of the positioner was created using Google SketchUp, a free 3D modeling tool. This initial design is shown in Figure 3.

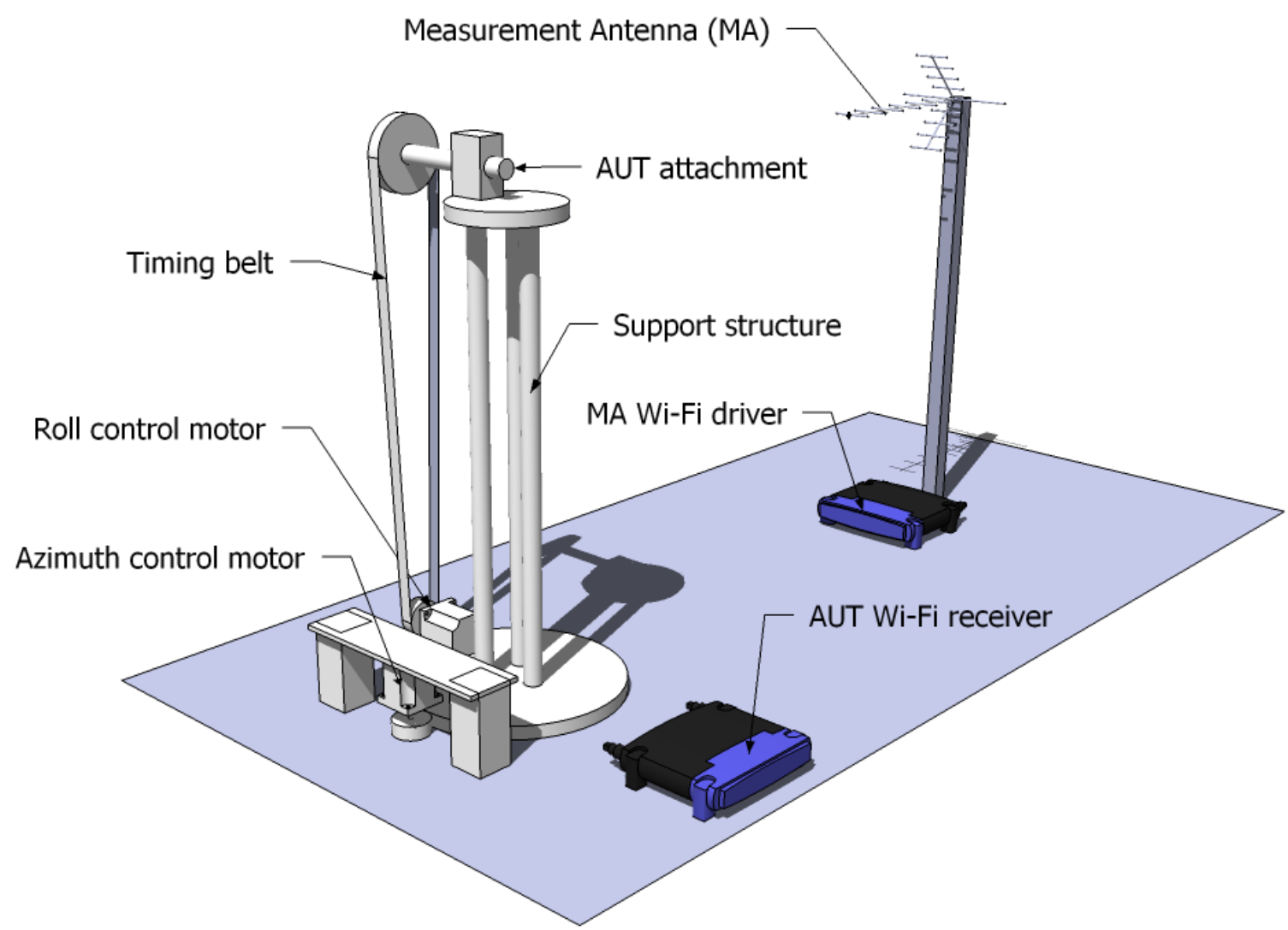

Figure 3: The initial design of the positioner created using Google SketchUp 
Yaw axis rotation is accomplished by using a turntable bearing, McMaster-Carr item 1797K2. This bearing is mounted on a plywood base, and a wood disk is attached to the top of the bearing. To attach this disk, additional holes are drilled in the base to allow access to the screws used to secure the disk to the bearing. The turntable bearing and plywood base is shown in Figure 4. The holes used to access the screws that secure the disk are visible under the mounting holes on the left and right edges of the bearing. The completed assembly, with the disk secured to the top of the bearing, is shown in Figure 5. This bearing employs a detent that could be used as a reference for the $\theta$ axis if desired. A large diameter bearing is selected to minimize wobble of the axis as it rotates. The disk is an 18 in (457 $\mathrm{mm})$ diameter wood round purchased from a home improvement store. The disk originally had a radiused edge. This radius was removed using a band saw at the UIC machine shop. The resulting disk is perfectly cylindrical. This is the only operation that required the use of machine shop equipment. This work was performed at no charge and is not included in the price of the system. 


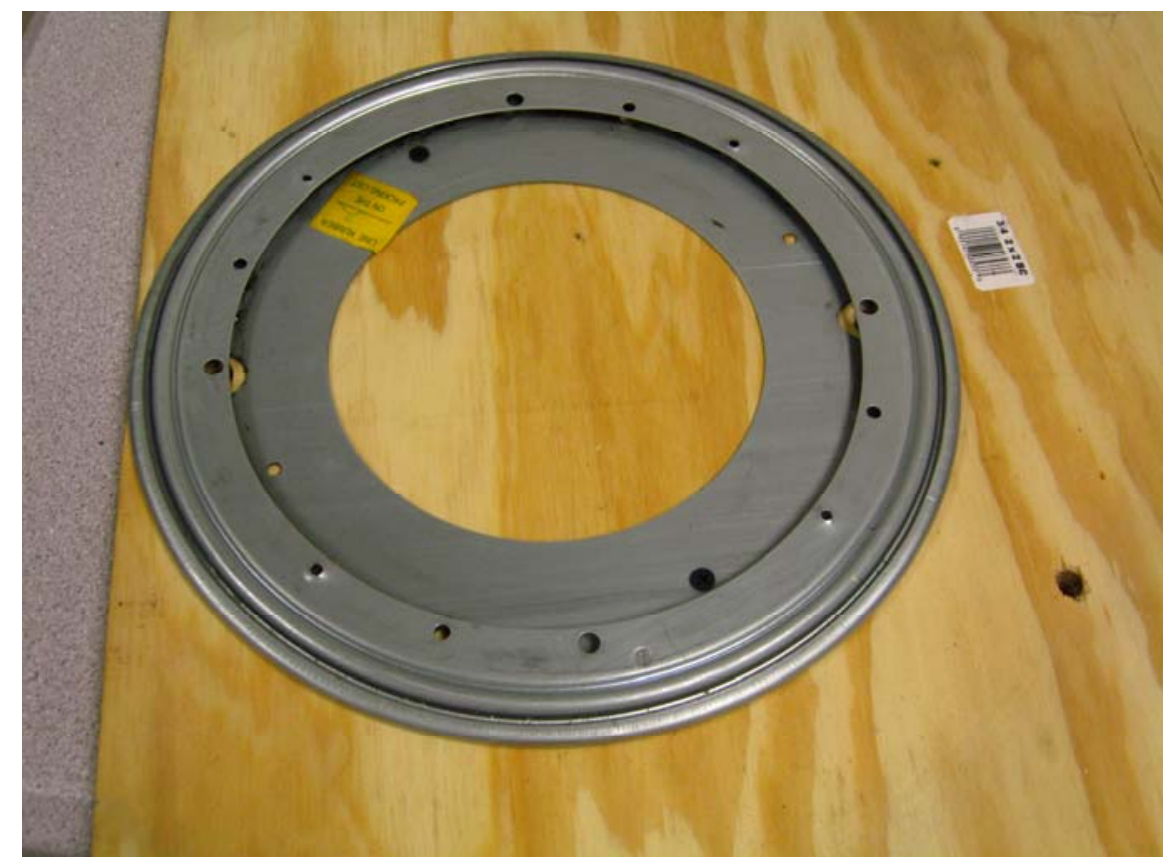

Figure 4: The turntable bearing attached to the plywood base. A disk is attached to the top of the bearing. Holes drilled through the base permit access to the screws used to retain the disk.

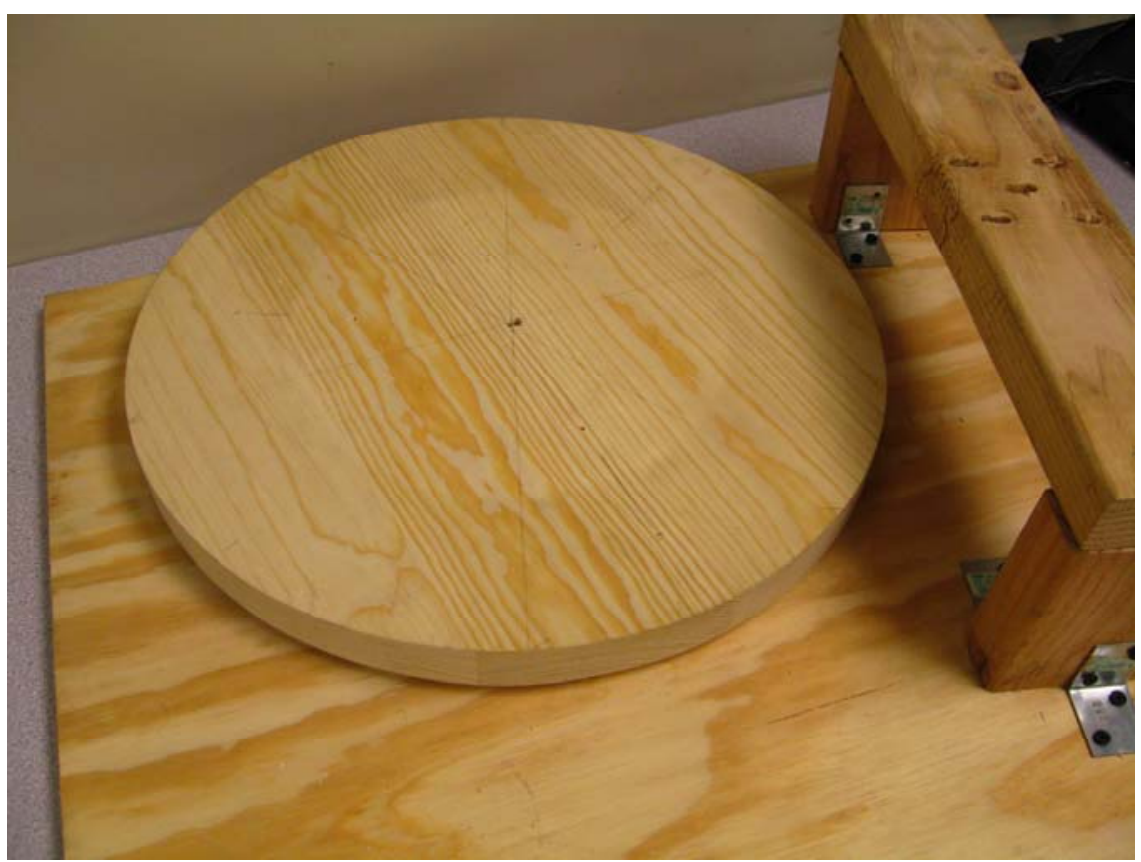

Figure 5: The yaw axis disk mounted on top of the turntable bearing. The support for the yaw axis motor has also been attached to the plywood base 
The antenna must be elevated above the yaw axis disk to allow the roll axis to be scanned. A column mounted on top of the yaw axis disk provides the necessary elevation and supports the roll axis. The column is constructed using three 1.25 in $(31.8 \mathrm{~mm})$ diameter oak dowels cut to 2 in $(50.8 \mathrm{~mm})$ in length, with wooden disks on each end of the dowels providing a support for the roll axis and an attachment to the yaw axis. The large diameter oak dowels provide exceptional rigidity, and the length is chosen to reduce the effect of ground reflections without making the system difficult to transport. The disks are made by laminating together 0.125 in $(3.18 \mathrm{~mm})$ thick wood disks purchased from a craft store. Several thin disks are glued together, and holes are drilled through this disk to accept the dowels. More thin disks are then glued to this disk, resulting in a single disk with sockets to accept the dowels. This is known as a mortise and tenon joint, and produces a strong and easy to assemble connection between the dowels and the disk. The column is located over the center of the yaw axis disk using a geometric construction, and is attached using wood screws.

The roll axis is attached to the top of the column. The axis consists of a section of 1 in (25.4 mm) diameter oak dowel. This dowel is held in place using a bearing block constructed from a section of 2 in $\times 4$ in (50.8 $\mathrm{mm}$ x $102 \mathrm{~mm})$ lumber. A hole is drilled in this block to accept the axis and a sleeve bearing. The block is attached to the top of the column using wood screws. To allow the dowel to rotate freely in the bearing block, a Delrin sleeve bearing, McMaster-Carr item 2705T44 is inserted into the bearing block. The bearing has a flange to locate the bearing in the block, and is slightly longer than the depth of the hole in the bearing block. This reduces the amount of deflection due to the 
tension of the roll axis drive belt. The axis is held in position using a Delrin clamp on shaft collar, McMaster-Carr item number 9410T38. A 6 in $(152 \mathrm{~mm})$ diameter pulley is created out of laminated disks, and a hole is drilled through the pulley to accept the dowel. To attach the pulley to the dowel, holes are drilled in an additional shaft collar to accept two \#10 size (4.83 mm) nylon screws. Corresponding holes are drilled through the pulley. The shaft collar is clamped onto the dowel, and nylon screws and nuts are used to affix the pulley to the shaft collar. To retain the belt on the pulley while still allowing the belt to be installed and removed easily, a flexible plastic flange is attached to the bearing. This flange is available at arts and crafts stores and is intended for use in flower arranging. The flange is attached using silicone adhesive. Nonmetallic bearings, shaft collars, and fasteners are used throughout this area to minimize electromagnetic scattering. To provide an attachment for the AUT, the dowel is cut longitudinally, so that a flat mounting surface is available. The completed roll axis assembly is shown in Figure 6. 


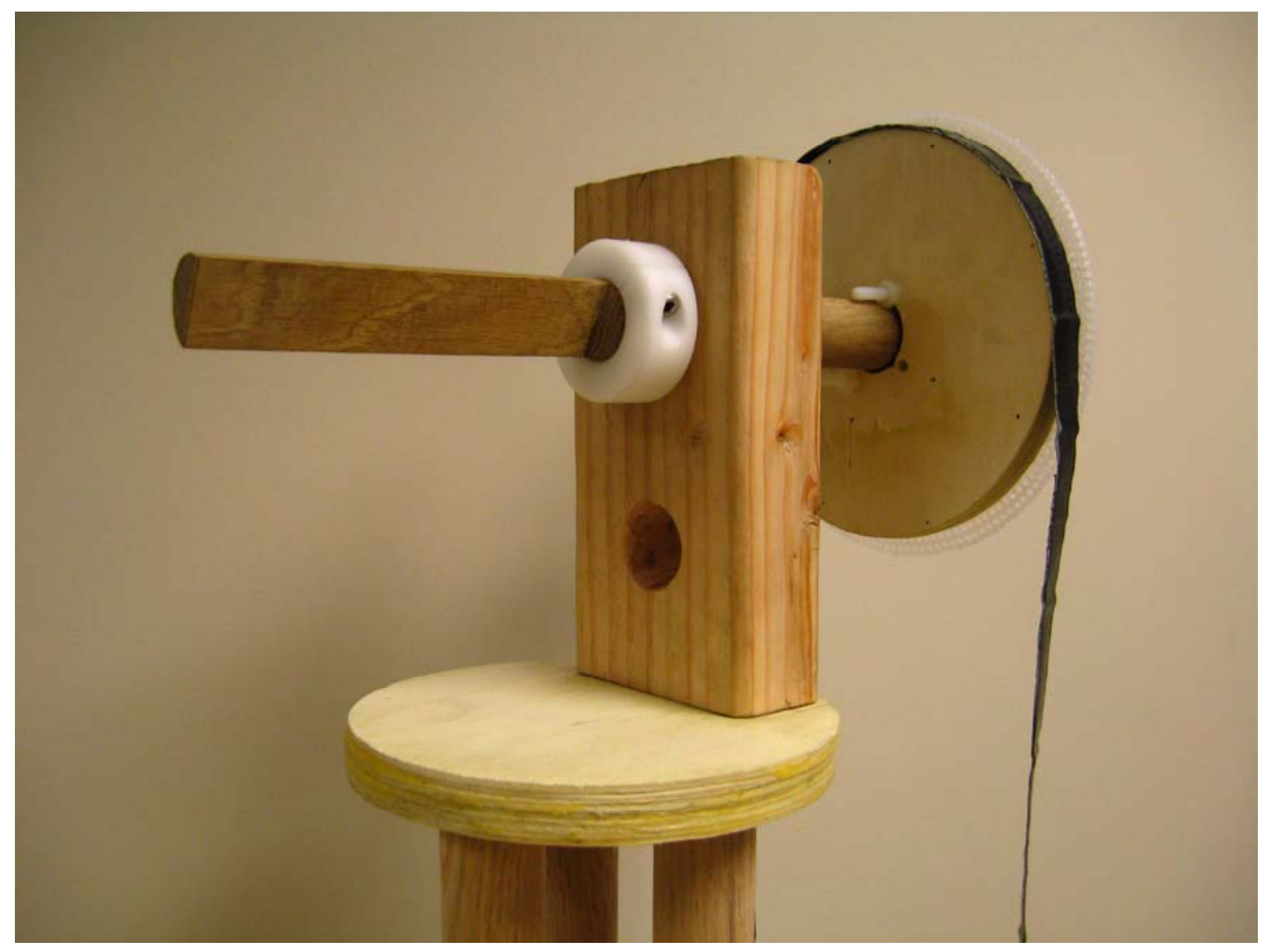

Figure 6: The completed roll axis assembly mounted on top of the column. The additional hole in the bearing block provides a path to route a coaxial cable

The positioner is now capable of movement in two axes, but a mechanism to drive each axis is still needed. Stepper motors are used because they allow the axes to be driven in known increments. To simplify the design of the system, commercially available components are used here as well. Stepper motors are widely used to build Computer Numeric Control (CNC) milling machines, and kits can be purchased that include everything necessary to interface a computer with a CNC milling machine. A kit produced by Xylotex is used here. The kit includes stepper motors, a DC power supply, and a driver board that allows the motors to be controlled by a parallel port interface. 
This kit costs \$310, an excellent value considering the time and expense required to develop a custom stepper motor controller.

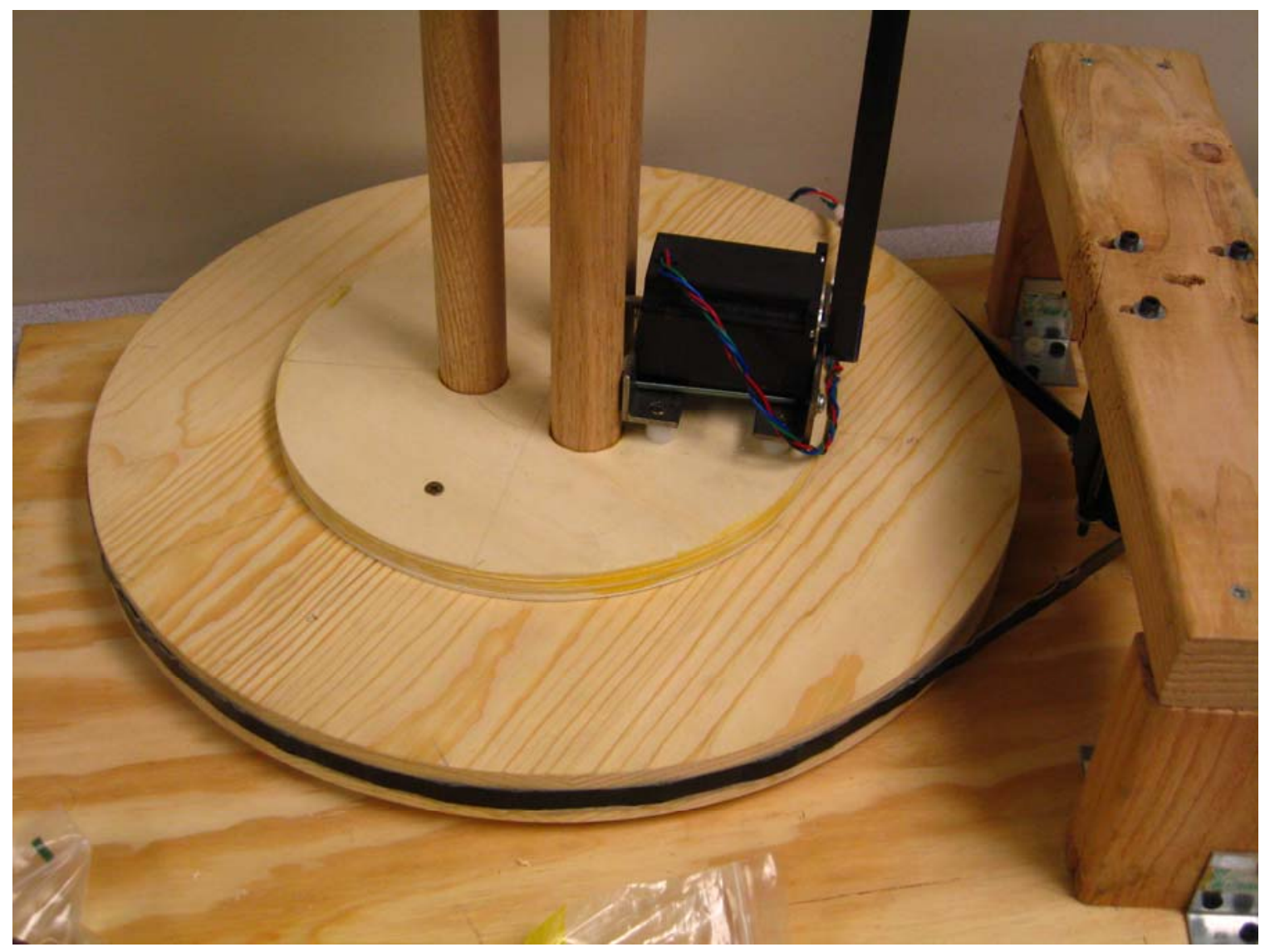

Figure 7: The stepper motors and their belt drives. The yaw axis motor is suspended from the brace at the right. The roll axis motor is fixed to the base of the column using angle iron brackets.

Each axis of the positioner is connected to a stepper motor using a belt drive, shown in Figure 7. The yaw axis was originally intended to be driven by direct contact between the yaw axis disk and a drive wheel on the motor. This method was abandoned when it was discovered that the disk would need to be perfectly circular; any irregularities would result in unreliable rotation. A belt drive does not require the pulleys 
to be perfectly circular. It also allows the metallic body of the motor to be located further from the antennas, reducing the effect of electromagnetic scattering. Each motor is fitted with a 1 in $(25.4 \mathrm{~mm})$ diameter drive pulley, McMaster-Carr item 2488K23. The pulley has a soft rubber surface to increase friction with the drive belt, and the small size allows each axis to be driven with a large gear ratio. The yaw axis motor is suspended from a brace assembled from 2 in $x 4$ in (50.8 mm x $102 \mathrm{~mm}$ ) lumber using long \#10 size (4.83 $\mathrm{mm}$ ) screws. This places its pulley in the same plane as the yaw axis disk. The screws are located in slots, allowing the motor to be repositioned to adjust the tension of the belt.

The roll axis motor is mounted on the yaw axis disk using brackets fabricated from a steel right angle profile. The profile is cut to the desired length, and holes are drilled to match the mounting holes on the face of the motor. The brackets are secured to the motor using long \#10 size $(4.83 \mathrm{~mm})$. Holes are drilled in the other face of the bracket to mount it to the yaw axis disk. The motor and bracket assembly is attached to the yaw axis disk with wood screws and nylon spacers. The spacers can be trimmed to adjust the tension in the belt if needed. The entire assembly is shown in Figure 8. 


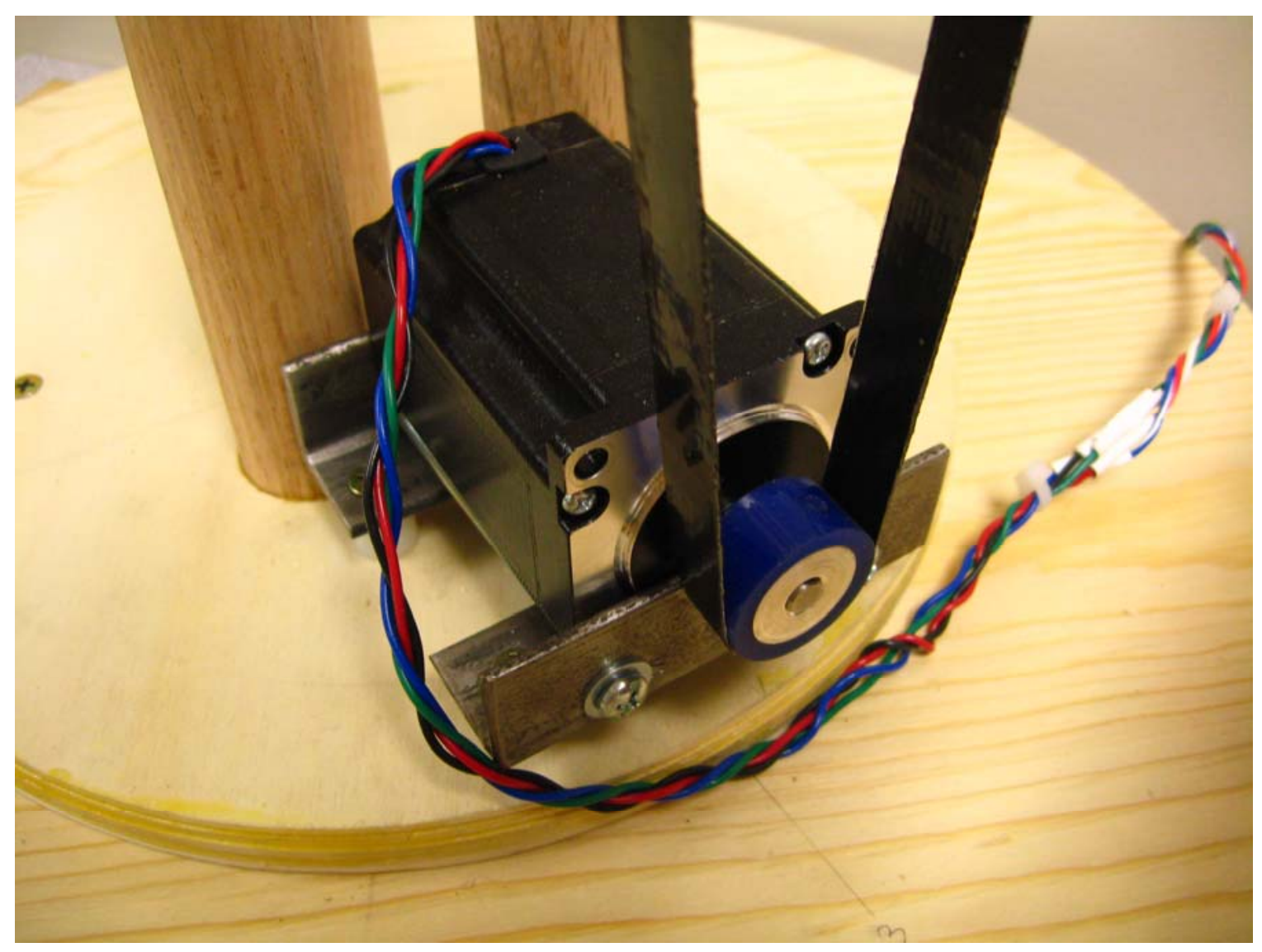

Figure 8: The roll axis motor and its custom fabricated mounting bracket, attached to the base of the column. Note the rubberized drive wheel fitted to the shaft of the motor

The belts are custom made using adhesive tape. Flat belts were purchased from McMaster-Carr, but the sizing guidelines provided in the catalog proved inaccurate. The indicated belt was far too small, and correctly sized belts were not available. Made to order belts could be purchased, but sufficient time was not available. Purchasing unassembled belting was also considered, but this requires a specialized tool to join the ends of the belting. Therefore, a method of fabricating the correct size belts was developed. This method utilizes layers of adhesive tape wrapped around a fixture used to ensure that the correct size is maintained. The fixture consists of pulleys clamped to a 
bench, with the distance between the pulleys adjusted to determine the size of the belt. To fit the pulleys, the width of the belt is .5 in $(12.7 \mathrm{~mm})$. A strip of duct tape is cut to this width and wrapped around the fixture to form the inside surface of the belt. Duct tape is used for its strength and higher friction surface. The inner layers of the belt are made from Scotch brand transparent tape, which is used because it could be purchased in the correct width. Several layers of Scotch tape are wrapped around the fixture before and additional strip of duct tape is cut to the correct width and wrapped around the fixture to form the outer surface of the belt.

The stepper motors are designed to rotate in increments of $1.8^{\circ}$ per step. The motor driver allows the motors to be operated in full, half-, quarter-, and eighth-step increments. To minimize vibrations, the motors are operated in eighth-step mode, resulting in a movement of $0.225^{\circ}$ per step. Although this causes the axes to rotate more slowly, this reduces the risk of a positioning error due to excessive vibration in the column. Accuracy is also improved by the use of large gear ratios for each axis. Based on the diameters of the pulleys used, the nominal gear ratios for the yaw and roll axes are 18:1 and 6:1, respectively. Due to variations from the nominal size of the pulleys, the actual gear ratio is determined empirically. At the nominal ratios, the positioner can be controlled with a maximum error of only $0.013^{\circ}$ in the yaw axis, and $0.038^{\circ}$ in the roll axis. This error is small enough that it would be unlikely to affect the results of a pattern measurement, and is comparable to the positioning accuracy of commercial systems.

The RF and positioner components are controlled by a computer running National Instruments LabVIEW instrument control software. LabVIEW is used because it allows 
users to develop Graphical User Interfaces (GUIs) to control a variety of hardware [21]. A customized interface is developed that allows users to perform antenna measurements and view results from a single window. This interface allows users to manually rotate the positioner and query the router's RSSI readings. The measurement procedure can also be fully automated. Users can select the number of positions to sample, which axis to rotate, and how many samples to record for each position. This allows users to scan an entire principal plane without intervention. When the scan is complete, users may export the results for further analysis using software such as MATLAB.

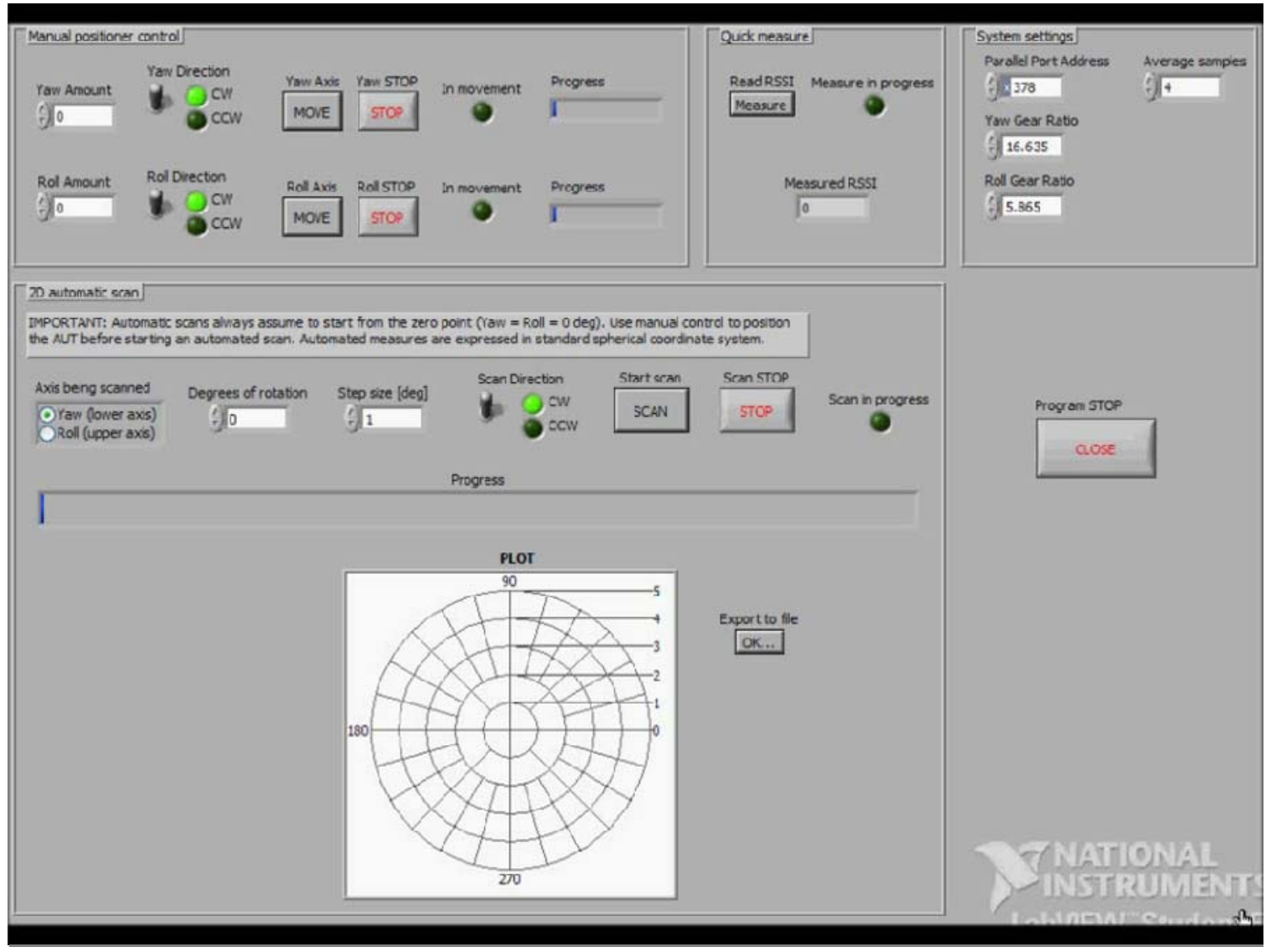

Figure 9: The LabVIEW GUI. Users can manually rotate the positioner, take RSSI measurements, set the port address and positioner gear ratios, and perform automated scans and view and export results 


\subsection{Calibration}

To confirm that the routers will provide sufficient dynamic range, a calibration procedure is performed. This is accomplished by comparing the measurements obtained with the routers to a spectrum analyzer measurement. The spectrum analyzer is assumed to be perfectly calibrated, and serves as a reference to compare the RSSI measurements against. The MA and AUT need not be known for this calibration, however, the test conditions must remain constant during the calibration procedure. The transmitting router is connected to the MA. A splitter allows the signal received by the AUT to be sent to both the receiving router and to a spectrum analyzer. Both the router and the spectrum analyzer should be connected to the splitter using identical cables. Using the dd-wrt interface, the transmitted power is varied over the entire allowable range in increments of $3 \mathrm{~dB}$. The received power as measured by the router and the spectrum analyzer is recorded for each transmitter power level. These measurements are shown in Figure 10. 


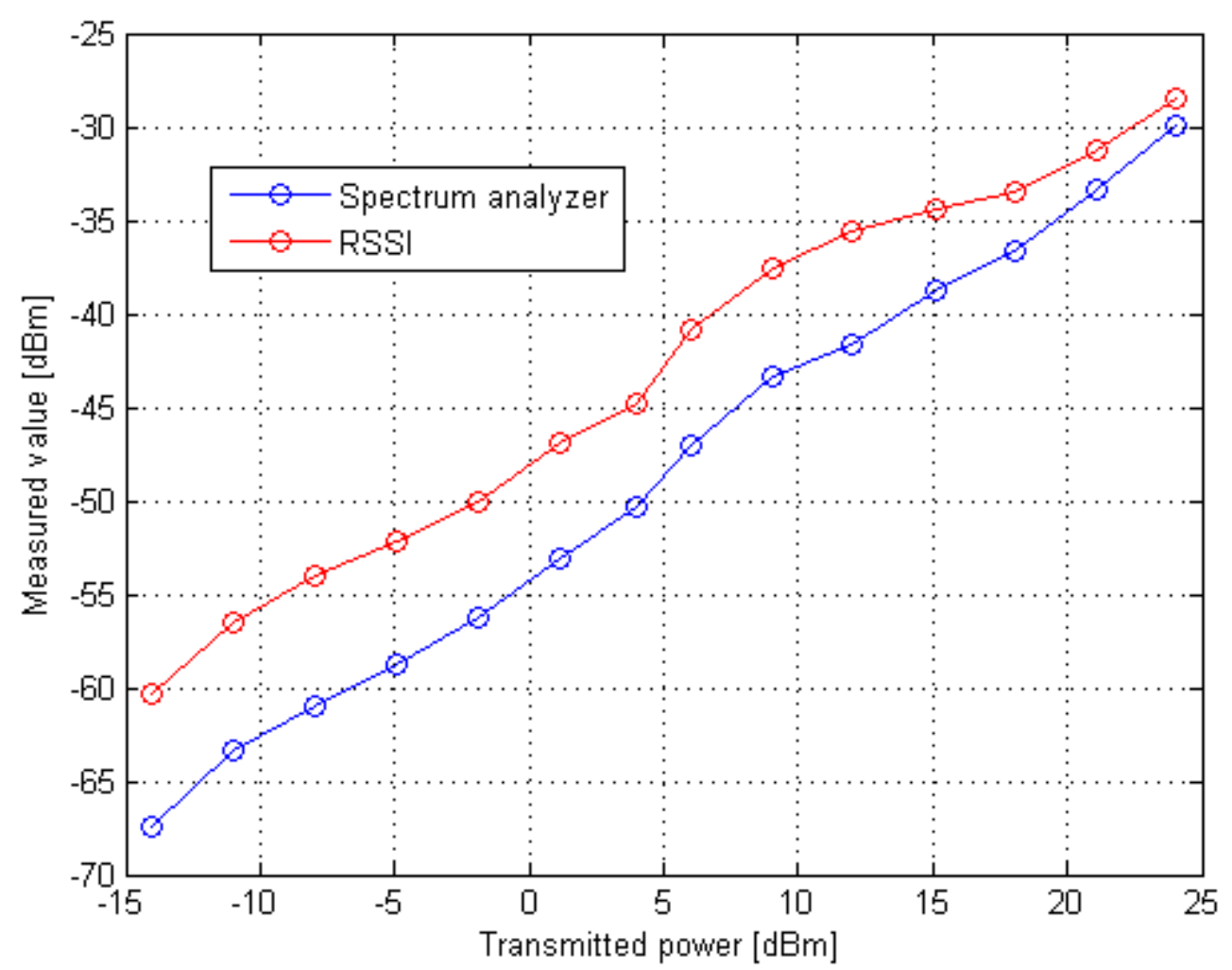

Figure 10: Received power measurements produced using a spectrum analyzer and the Wi-Fi router. Transmitted power is reported by the transmitting router

The spectrum analyzer is assumed to be perfectly linear. Therefore, any variation in the power measured by the spectrum analyzer is assumed to be caused by the transmitting router. The transmitting router may not produce the amount of power selected in the ddwrt control panel. However, because the difference between the spectrum analyzer and router measurements will be used, this variation does not affect the results. When the spectrum analyzer result is subtracted from the RSSI value, the difference in each reading for each power level is obtained. This difference is shown in Figure 11. In this figure, the independent axis indicates the RSSI measurement obtained using the router. The 
dependent axis is the difference between the RSSI measurement and the spectrum analyzer measurement. The interpolated value of this difference as determined by a polynomial estimator is also shown.

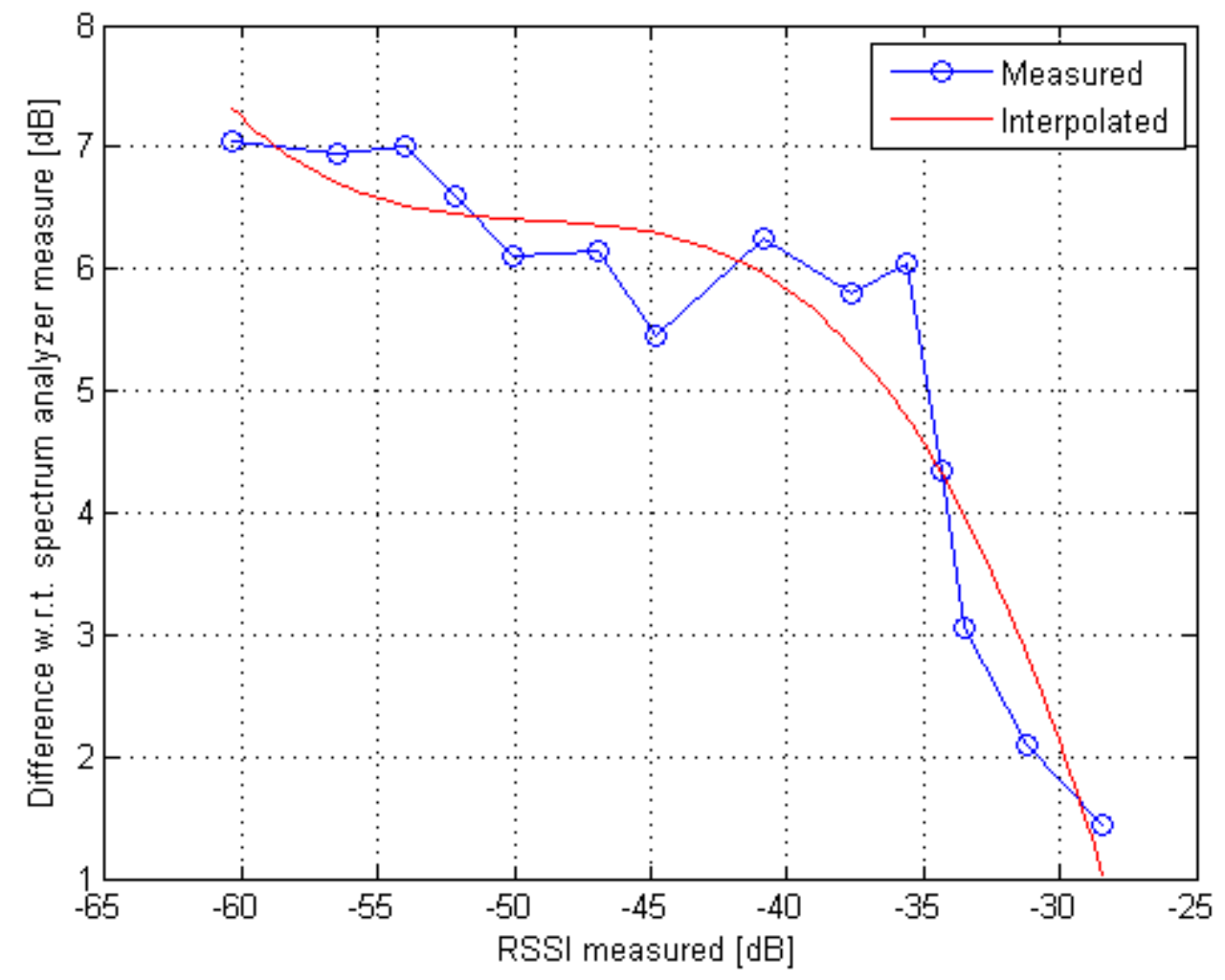

Figure 11: Difference between the RSSI value returned by the router and the spectrum analyzer measurement as a function of the RSSI obtained from the router

In general, the difference is small for high levels of received power and increases when the signal strength decreases. This difference varies by less than $1 \mathrm{~dB}$ for RSSI values in the approximate range of -61 to $-47 \mathrm{~dB}$, resulting in a linear range of approximately $14 \mathrm{~dB}$. The measured differences are interpolated using a third order 
polynomial estimator, which allows a correction coefficient to be computed from the following function:

$$
C=-0.00057 \operatorname{RSSI}_{d B}^{3}-0.0836 \operatorname{RSSI}_{d B}^{2}-4.1314 \operatorname{RSSI}_{d B}-61.863
$$

This correction coefficient is applied to the RSSI values obtained from the router, allowing the RSSI values to match the power measurements obtained from the spectrum analyzer. Applying this correction minimizes the difference between the RSSI obtained with the routers and the value one would expect to see when using a spectrum analyzer. The linear range of the router is extended to the range over which the calibration is performed, approximately $-61 \mathrm{~dB}$ to $-28 \mathrm{~dB}$. Applying the calibration extends the linear range from $14 \mathrm{~dB}$ to $33 \mathrm{~dB}$.

Performing this calibration against an instrument allows the performance of the router to be verified and enhanced, but is not essential. As is demonstrated by the pattern shown in Figure 2, it is possible to produce reasonable results without prior calibration of the routers. If the received power remains within the linear range identified earlier, the impact of the calibration is minimal. Finally, because the routers are mass produced, it may also be possible to assume that all routers of this model and version number will behave similarly. This would allow the same calibration to be reused if a spectrum analyzer is not available. However, repeating this procedure whenever a different router is used provides the highest degree of certainty that sufficient dynamic range will be available. 
The routers provide RSSI as an integer value between -100 and 0 . Representing the received power with an integer introduces quantization error. This error will always be less than half the step size of the RSSI values. In this case, the error will always be less than $0.5 \mathrm{~dB}$. This error is assumed to be uniformly distributed within this range [22]. The quantization error can therefore be represented as a uniform random variable between $-0.5 \mathrm{~dB}$ and $0.5 \mathrm{~dB}$. This satisfies the requirements of the competition. However, the quantization error can be further reduced using dithering. In the absence of noise, repeating a measurement should result in obtaining the same results. For example, attempting to measure the value of $-4.8 \mathrm{~dB}$ will always result in a reading of $-5 \mathrm{~dB}$. However, by introducing noise with an amplitude similar to the magnitude of the quantization error, the value of $-4.8 \mathrm{~dB}$ plus a uniformly distributed error is measured [23]. This value is a uniformly distributed random variable with a mean of $-4.8 \mathrm{~dB}$. When a large number of measurements is made and averaged, the law of large numbers implies that the result will converge on the mean of the random variable. To exploit this effect, the user has the option to automatically average several samples whenever a measurement is taken. The user can specify the number of samples to use, although several samples are required to demonstrate convergence. During pattern measurements no effort is made to introduce noise beyond what is already present in the system. However, when performing an absolute gain measurement, additional "noise" is introduced by repeating the measurement at different power levels. This offset has the same effect as introducing noise, and should result in obtaining the same amount of gain at all power levels. 


\section{EXPERIMENTAL RESULTS AND ANALYSIS}

\subsection{Test Setup}

The performance of the system was evaluated by measuring the far field patterns of a microstrip patch array, a dipole, and a Yagi antenna. The measurements were conducted outdoors to minimize the effect of reflections, in the area behind a lab member's home. The test setup is shown in Figure 12. The test area was approximately $5 \mathrm{~m} \times 5 \mathrm{~m}$ square, with the main lobe of the MA pointed at a wooden fence. The positioner and MA were placed on tables to elevate them above the ground. The ground consisted of a grass lawn and a concrete surface. The positioner was placed over the grass region to minimize the effect of scattering on the measurement. The relative permittivity of soil, $\varepsilon_{r}$, is approximately 3, compared to 4.5 for concrete. Electromagnetic energy traveling through air would therefore be expected to be more strongly reflected when encountering concrete. 


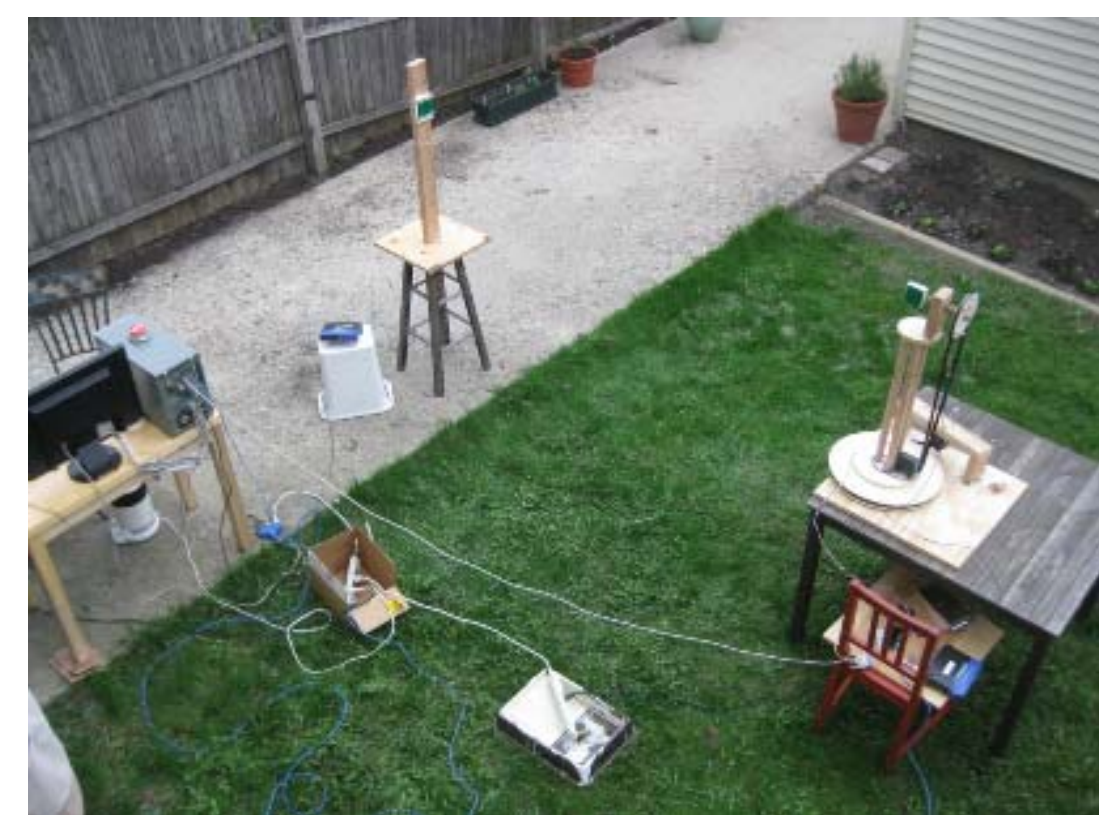

Figure 12: The test setup used to evaluate the system, showing the positioner, routers, and controlling computer located in an outdoor environment

A $360^{\circ}$ scan in $5^{\circ}$ increments was made in both principal planes of each antenna. To reduce quantization errors, eight samples are averaged in each position. To validate the test results, the far fields of each antenna were simulated using FEKO. These simulation results will be described in further detail below. This provides a reasonable approximation of the far field pattern of the patch array. The measured values are normalized, and superimposed with the simulation results to facilitate comparison.

\subsection{The Test Antennas}

The patterns of three different types of antennas were measured. Two were intended for use in Wi-Fi systems, and were purchased inexpensively from an online retailer. The third antenna, a $3 \mathrm{GHz}$ half wave dipole, was available in the lab. The cost of this antenna was not included in the cost of reproducing the system, as it is not strictly 
necessary to reproduce the system. It is used simply to provide additional test results. Testing a dipole is especially desirable because its pattern is well known, and can be determined analytically. This particular dipole is electrically small when operated at WiFi frequencies. Electrically small dipoles will exhibit less gain, but the shape of the pattern will remain similar. Simulation results confirm this, and when this antenna is used as a gain standard, its gain is estimated to be slightly lower than a correctly sized half-wave dipole.

The two Wi-Fi antennas are implemented on printed circuit boards (PCBs) and are fed using coaxial cables with SMA connectors. One is a five element Yagi design. The Yagi features a single driven element, with additional parasitic elements designed to increase the gain of the antenna. This Yagi incorporates a single reflector element, and three director elements.

The other Wi-Fi antenna is a planar array. The array elements are microstrip patches, and are fed using microstrip. The back of the antenna is covered with a metallic ground plane.

The patterns of these antennas are not provided by the manufacturer. To determine if the pattern has been measured accurately, the test results are therefore compared to simulation results. These simulation results are obtained by designing antennas of identical geometry in FEKO. Due to the difficulty of creating a model that exactly replicates the feed structure of the array antenna, this antenna is simulated as having a separate feed for each element. Each of the four feeds is fed with a phase offset determined by measuring the antenna's microstrip feeds. The resulting model should 
produce a pattern identical to one produced with a model that replicates the feed used on the actual antenna. To further validate the results, the simulation results are compared to analytical results produced for the dipole and microstrip array. The analytical solution of the dipole pattern can be obtained simply. The microstrip array pattern is obtained by finding the array factor of this array, and multiplying it by the pattern of an individual microstrip element.

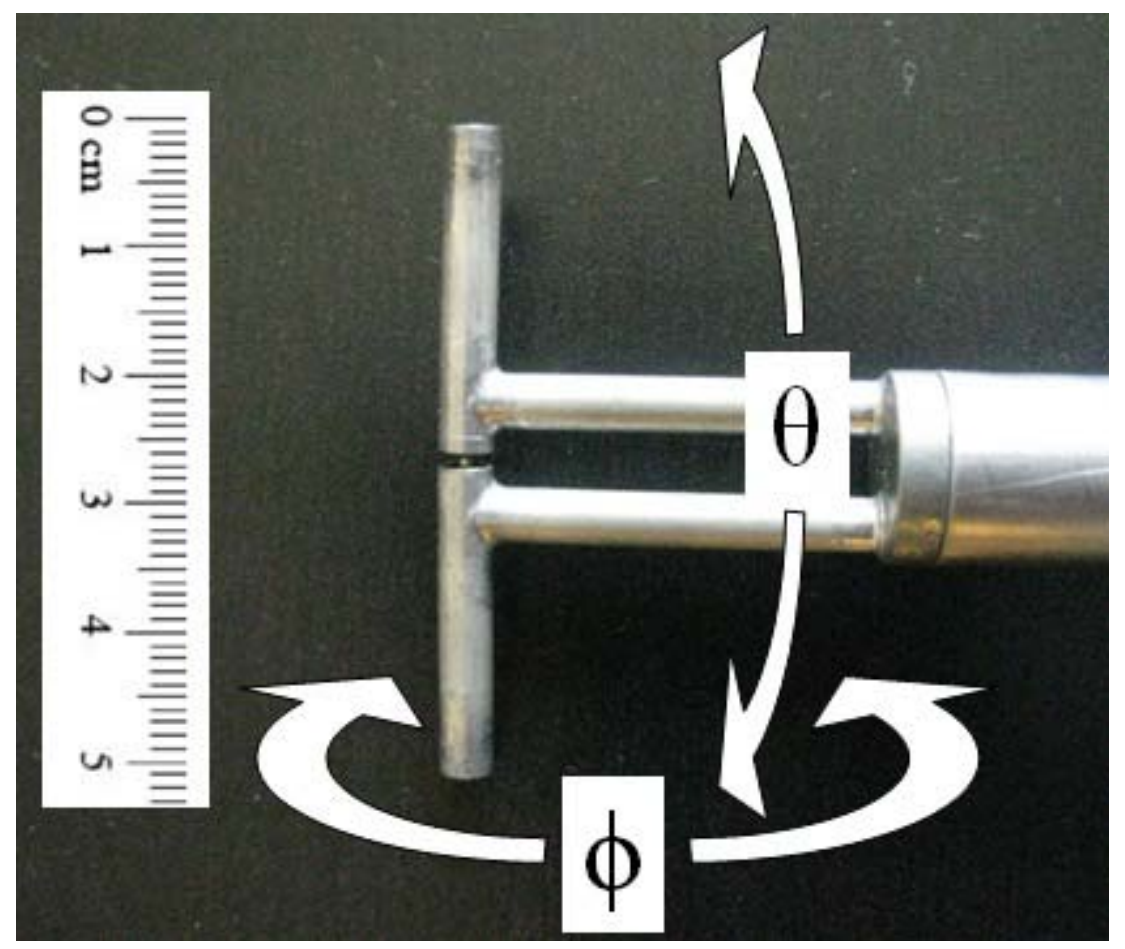

Figure 13: The $3 \mathrm{GHz}$ half wave dipole. The directions used in the scan are indicated 


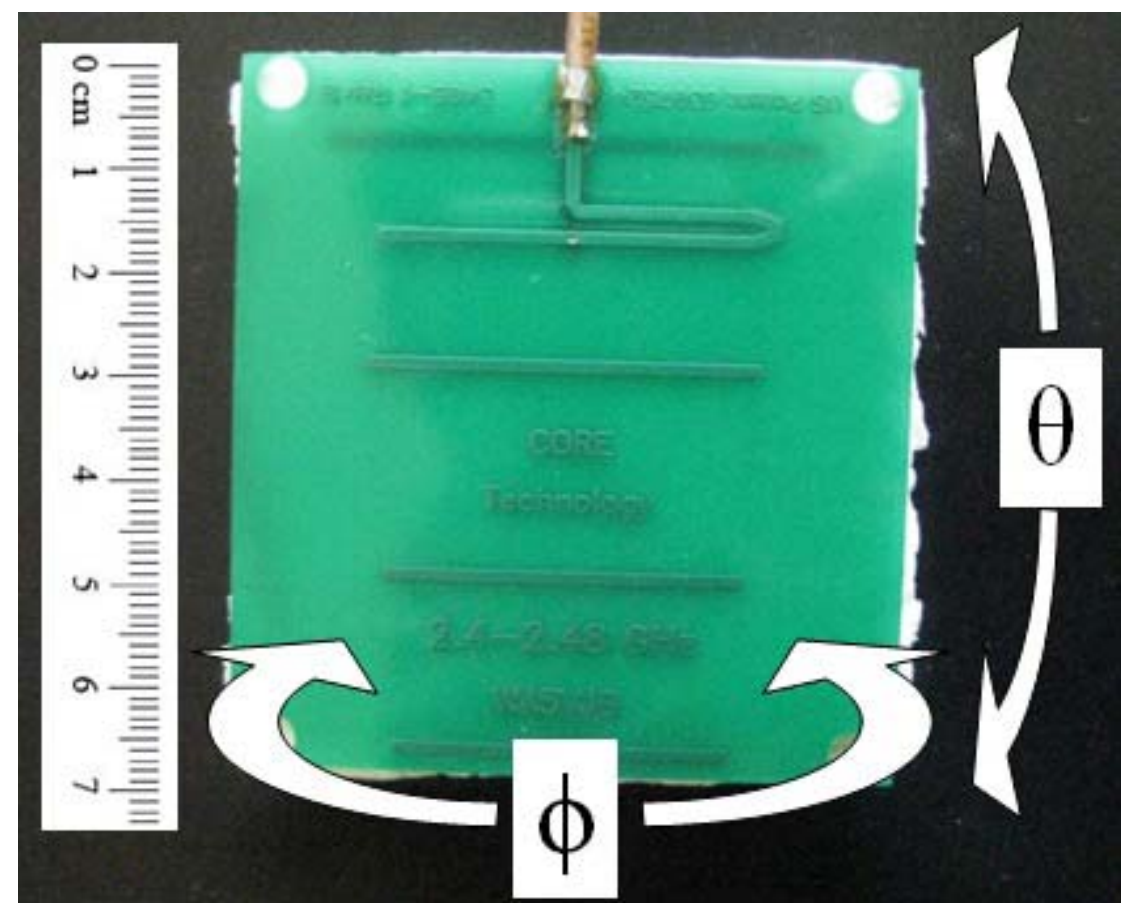

Figure 14: The Wi-Fi Yagi antenna. The directions used in the scan are indicated

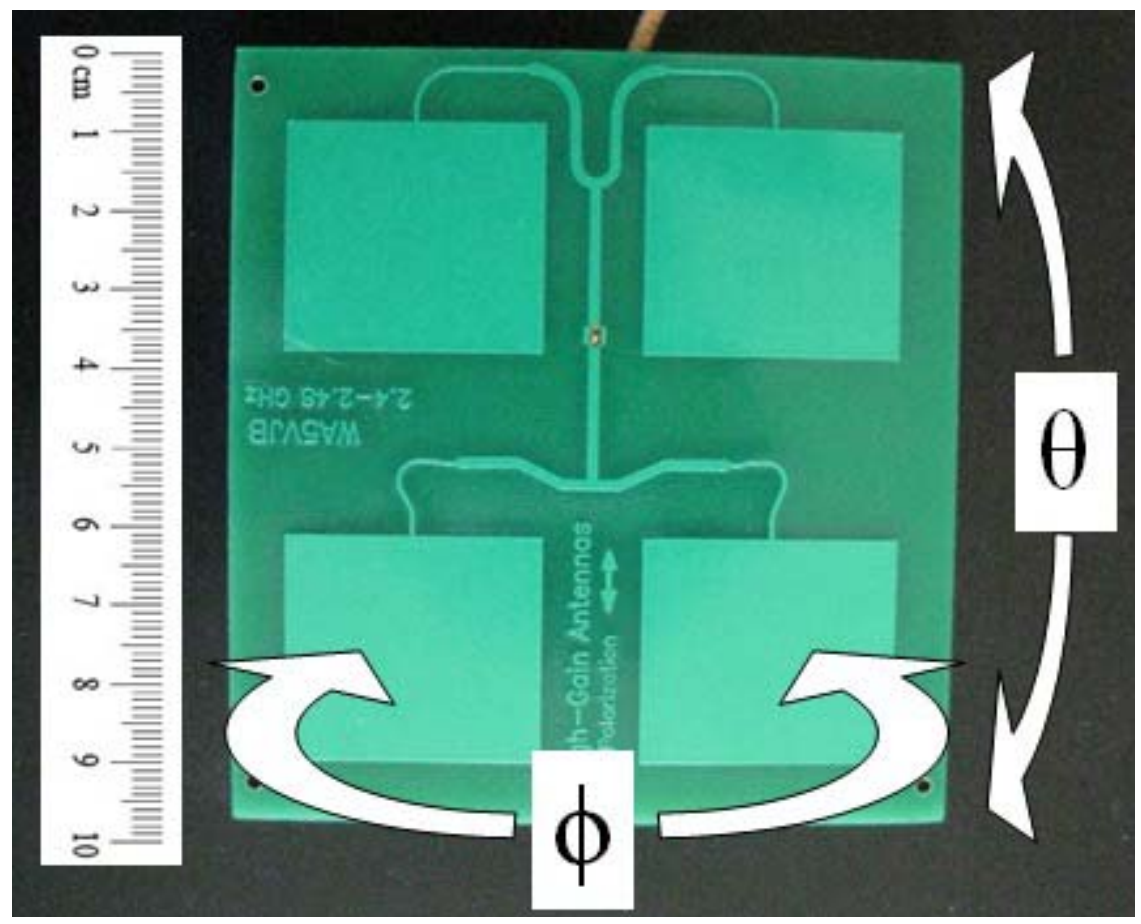

Figure 15: The microstrip patch array antenna. The directions used in the scan are indicated 


\subsection{Test Results}

\subsubsection{The Half-wave Dipole}

The pattern of the dipole is well known and can be determined analytically with relative ease. By determining this pattern analytically, and numerically with FEKO, an excellent reference to compare measured results against is obtained. Analytical and numerical results indicate that in the $\theta$ plane, the half-wave dipole is expected to have a toroidal pattern. The pattern in this plane produced using FEKO is shown in Figure 16, as are the measured results. The measured results clearly show this toroidal pattern, with the expected nulls at $90^{\circ}$ and $270^{\circ}$. While some irregularities are present, the distinct pattern of the dipole is readily apparent. In the $\varphi$ plane, analytical and simulation results indicate that the half-wave dipole will have a perfectly circular pattern. The measured result and simulation results for this plane are shown in Figure 17. The measured pattern is not perfectly circular, although some irregularities are expected in any experimental data. No distinct lobes are present, so in this plane, the pattern can still be assumed to be omni-directional. By comparing these measured results to the well defined pattern of the half-wave dipole, it can be concluded that this system provides reliable pattern measurements. 


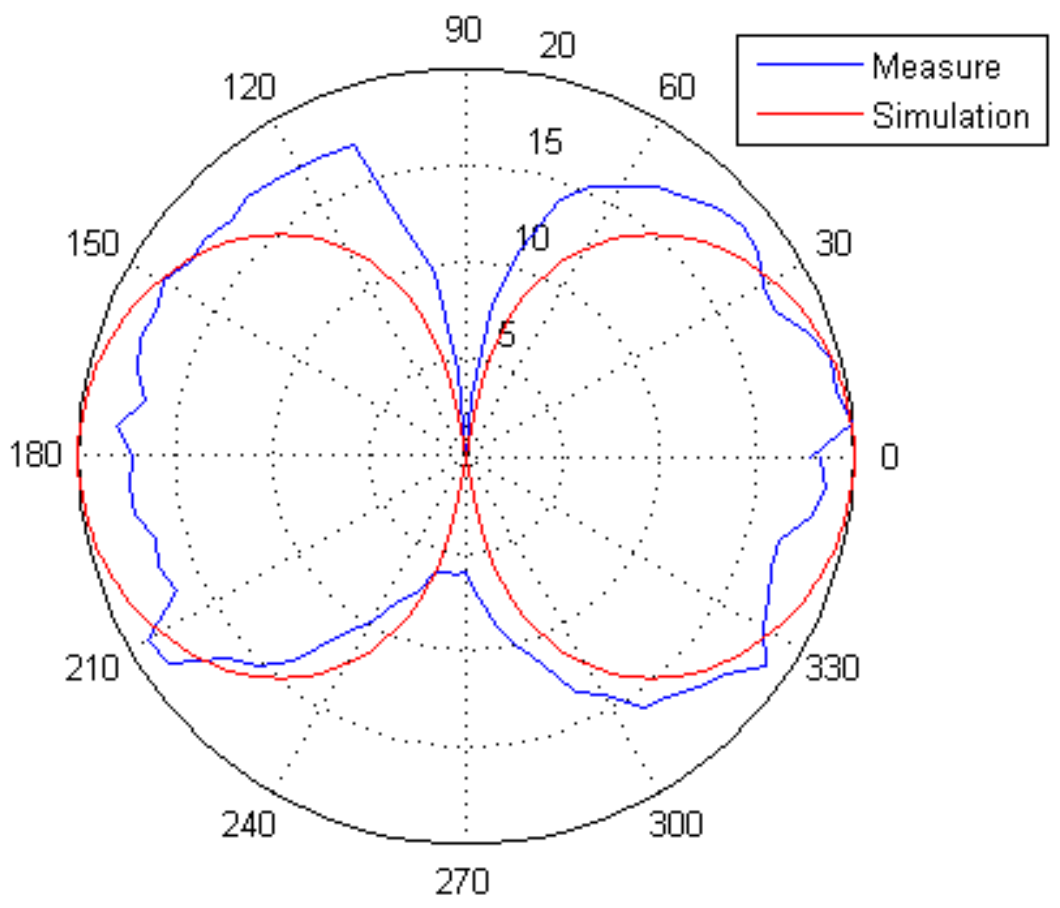

Figure 16: Normalized $\theta$ plane pattern of a dipole obtained using the Wi-Fi system. The simulated behavior of this dipole is superimposed over the measured results 


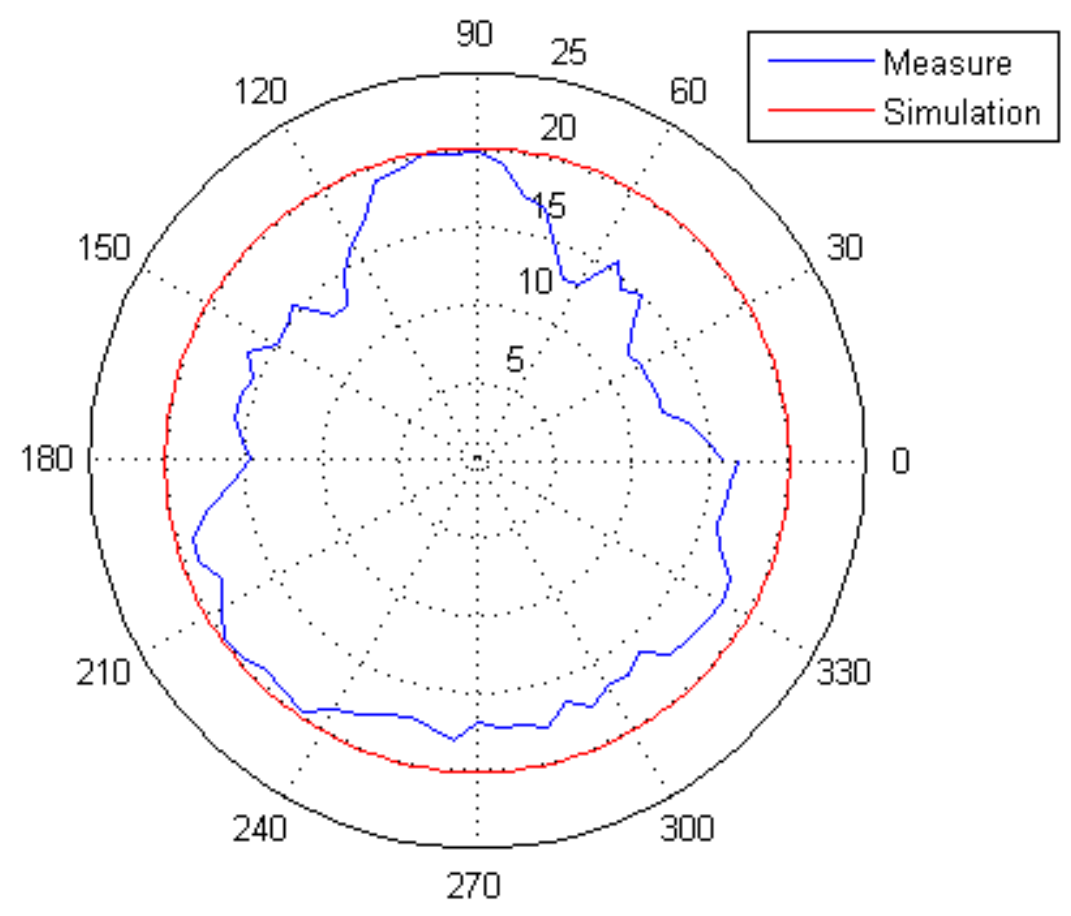

Figure 17: Normalized $\varphi$ plane pattern of a dipole obtained using the Wi-Fi system. The simulated behavior of this dipole is superimposed over the measured results

\subsubsection{The Microstrip Patch Array}

Based on the simulation results, the patch array is expected to produce a single, large lobe. This is confirmed by an analytical computation of the array factor of a 2 by 2 planar array, multiplied by the pattern factor of an individual microstrip antenna element. The simulation is also performed using a modified feed structure, and an infinite substrate. These modifications are performed due to technical limitations of the simulation software. The changes to the feed should not affect the pattern of the array. However, because the simulation is performed with an infinite substrate, the actual results will show some variation due to diffraction effects from the finite substrate. The measured results in the $\theta$ plane are shown in Figure 18, along with the simulation results. 
The measured results clearly show a single large lobe, with a small back lobe. Small side lobes are also present; these may be the results of the finite substrate of the array. Due to the symmetry and recurrence of these features in multiple measurements, they are likely a physical phenomenon and not spurious. They are also visible in the $\varphi$ plane, as seen in Figure 19. The large main lobe in the $\varphi$ plane coincides well with the simulation results, and the small side lobes are present here as well, further proof that they are legitimate.

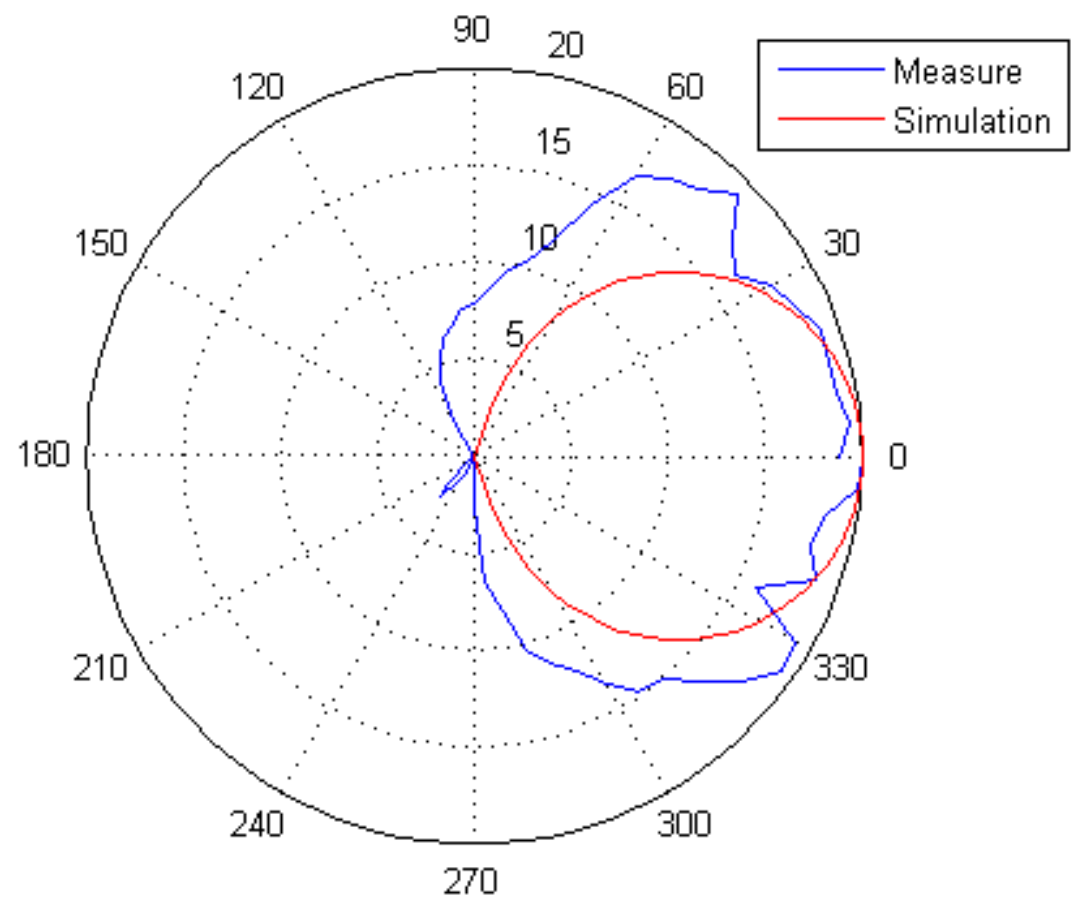

Figure 18: Normalized $\theta$ plane pattern of a microstrip array obtained using the Wi-Fi system. The simulated behavior of this antenna is superimposed over the measured results 


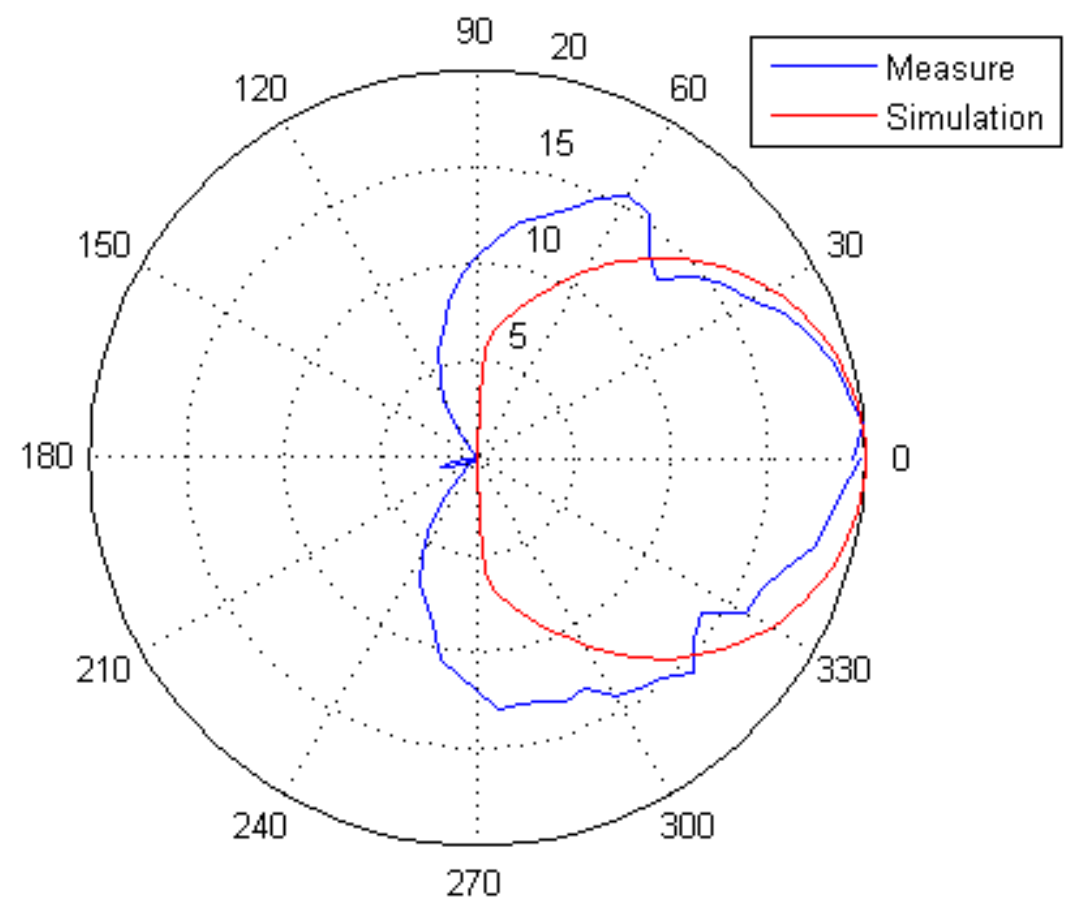

Figure 19: Normalized $\varphi$ plane pattern of a microstrip array obtained using the Wi-Fi system. The simulated behavior of this antenna is superimposed over the measured results

The effect of reflections off the ground was also observed in the microstrip array pattern measurements. These reflections are manifested by the distinctive asymmetrical lobes in the $\theta$ plane pattern of the array. These lobes occurred when the broadside lobe and the backlobe were pointed towards the ground. The oversized lobes seen at $300^{\circ}$ and $145^{\circ}$ in Figure 20 are very noticeable when compared to the pattern in Figure 18 . The results shown in Figure 18 are obtained while scanning the yaw axis, while those shown in Figure 20 are obtained while scanning the roll axis in the clockwise direction. To confirm that this effect was due to reflections off the ground, the scan was repeated while rotating the roll axis in the counterclockwise direction. The results are shown in Figure 21. Because the $0^{\circ}$ position is always assigned to the start of the scan, with the angle 
increasing as the scan progresses, the same pattern should be reflected by $180^{\circ}$ when the scan direction is reversed. This is indeed the case. Figure 21 is a near perfect reflection of Figure 20.

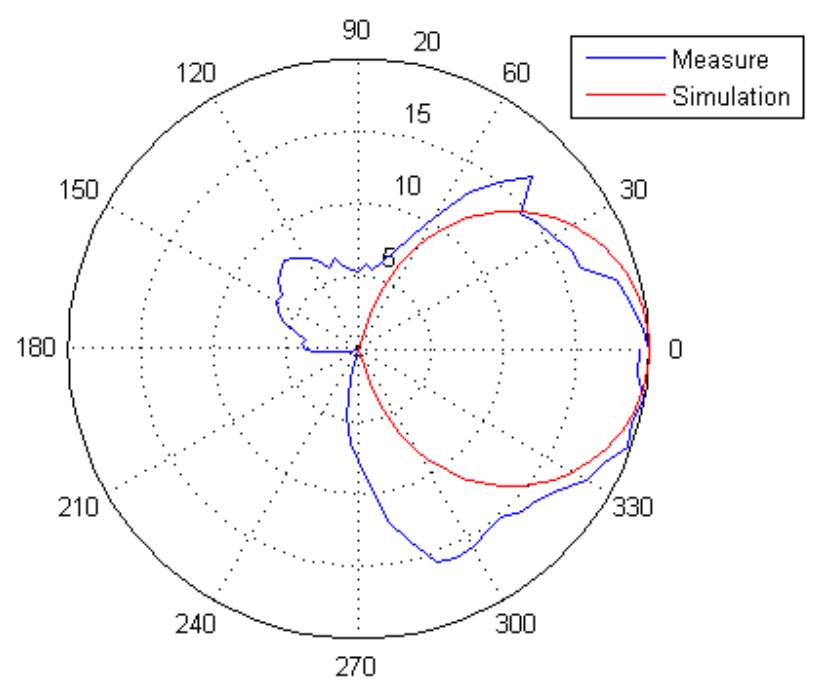

Figure 20: Normalized $\theta$ plane pattern of a microstrip array obtained using the Wi-Fi system scanning the roll axis in the clockwise direction.

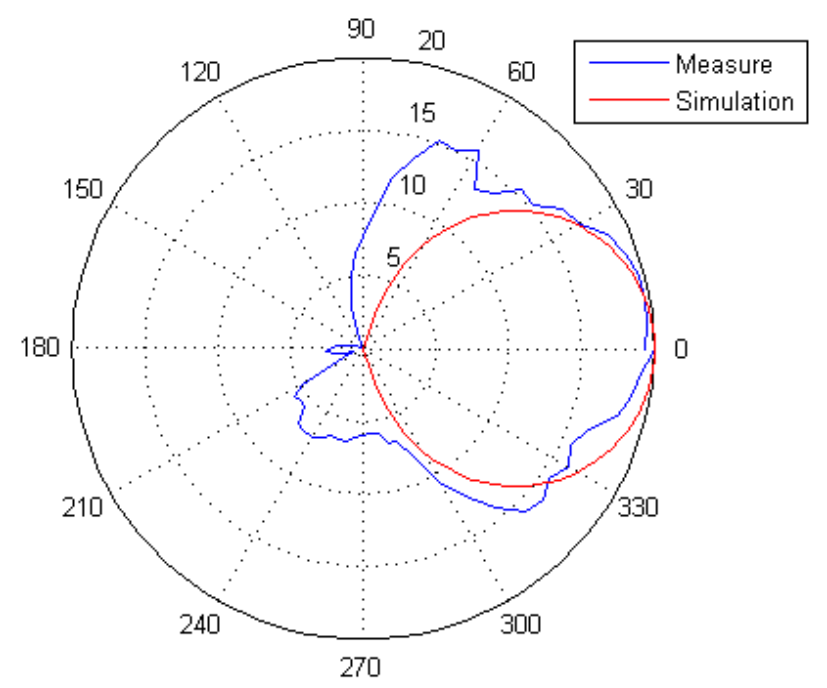

Figure 21: Normalized $\theta$ plane pattern of a microstrip array obtained using the Wi-Fi system scanning the roll axis in the counter-clockwise direction. 


\subsubsection{The Yagi Antenna}

Unlike the dipole and the patch array, an analytical computation cannot be made to confirm the simulation results of the Yagi antenna. However, the previous measurements have been very representative of the results obtained analytically and through simulation. Therefore, the simulation results alone will be used as a reference to compare the experimental results to. The entire Yagi antenna is simulated, including the feed structure, on a finite dielectric substrate. In the $\theta$ principal plane, a large main lobe is expected, along with a smaller back lobe. The measured results in Figure 22 show a large main lobe that coincides very well with the simulation results. A small additional lobe is present in the measured results. This may be the result of scattering caused by the coaxial feed cable mounted on the back of the antenna. 


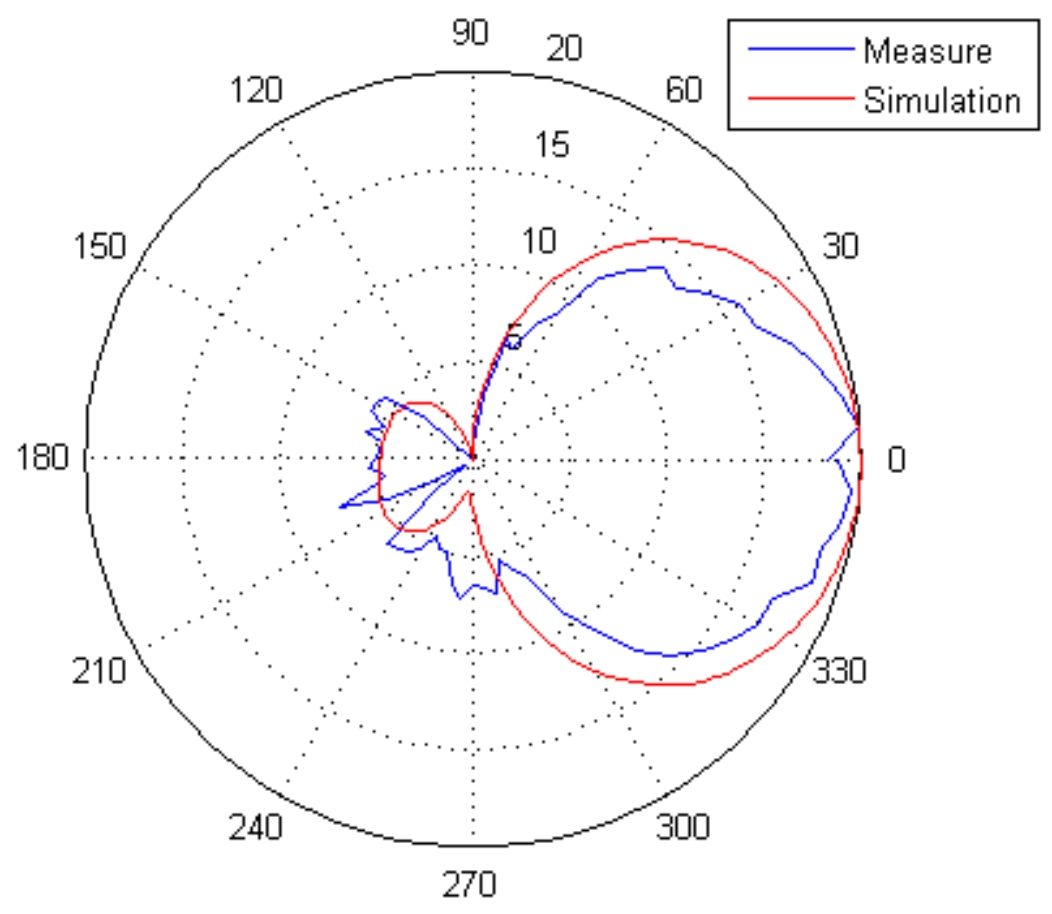

Figure 22: Normalized $\theta$ plane pattern of a Yagi antenna obtained using the Wi-Fi system. The simulated behavior of this antenna is superimposed over the measured results

\subsection{Gain Measurements}

To evaluate the system's ability to produce absolute gain measurements, the gain of the Yagi and microstrip array antennas is measured. The experimental results will be compared against the advertised gain of these antennas- $8.5 \mathrm{dBi}$ and $11.5 \mathrm{dBi}$, respectively. The measurements will be conducted using the gain transfer method described earlier. The absolute gain methods are not used because they require knowledge of the transmitted power, $P_{t}$. While the dd-wrt firmware allows the approximate transmitted power to be set, the routers do not provide a transmitted power measurement that is comparable to RSSI. Because the absolute gain methods depend on 
the ratio of received power to transmitted power, they do not yield valid results in this system.

A half-wave dipole has a well defined and consistent gain, making it suitable for use as a standard gain antenna. The other available antennas have gain values that are highly dependent on the design of the antenna. This makes them unsuitable for this purpose, where gain needs to be known a priori. The typical Standard Gain Horn is also not suitable for this measurement. The far-field distance of an antenna is determined by the following relationship:

$$
r \geq \frac{2 D^{2}}{\lambda} .
$$

For a given wavelength, the far-field distance depends on the square of the largest dimension of the antenna's aperture. Standard Gain Horns designed to operate at 2.4 GHz require a large aperture. For example, the Satimo SGH-170 Standard Gain Horn is designed to operate from $1.7 \mathrm{GHz}$ to $2.6 \mathrm{GHz}$ [24]. This antenna has aperture of $373 \mathrm{~mm} \times 277 \mathrm{~mm}$. Operating at $2.4 \mathrm{GHz}$ in free space results in a wavelength of $0.125 \mathrm{~m}$. For this wavelength, an antenna of this size has a far-field distance of at least $2.2 \mathrm{~m}$. The restrictions of the test environment do not allow the far-field condition to be attained for an antenna of this size. The size and weight of this antenna also make it incompatible with the positioning system. Finally, a true Standard Gain Horn is very expensive. Because the traditional Standard Gain Horn is not suited for this application, an alternative SGA is used. A half-wave dipole is expected to have a gain of $2.15 \mathrm{dBi}$. However, the dipole at hand was designed to operate at $3 \mathrm{GHz}$, making it slightly smaller 
than a half-wave length at $2.4 \mathrm{GHz}$. Electrically small dipoles exhibit less gain than fully sized ones. Based on our simulation results, we estimate that this dipole has $2 \mathrm{dBi}$ of gain.

If additional antennas with known gain are available, these may also be used for gain transfer method measurements. If the gain of an additional antenna can be determined using the dipole as a standard gain antenna, the additional antenna may be used to replace the dipole, if desired. This attribute of the gain transfer method may be useful for others attempting to reproduce this system who may not have access to a similar dipole. In this case, after determining the gain of the Yagi antenna, this widely available antenna can be used as a gain standard under the assumption that its gain will not vary between specimens.

As in a pattern measurement, the standard gain antenna and the AUT are both illuminated by a MA. The received power is measured for both the standard gain and the AUT. When the Yagi antenna is used as the AUT, the microstrip array is used as the MA. The Yagi antenna is used as the MA when the microstrip array is used as the AUT. When measuring the gain of the Yagi in our tests the patch array was used as the MA.

To reduce the effects of quantization noise, several measurements are made and averaged together, with the assumption that the results will converge on the actual value. The transmit power is varied as well, and the results obtained with different power levels are averaged. Although a correction coefficient is used to improve the linearity of the receiver system, averaging together measurements made at different power levels should further reduce the effects of non-linearity in the transmitted power levels. Transmit 
power is varied in increments of $3 \mathrm{~dB}$ over the entire available range. Received power is measured at each power level for both the standard gain antenna and the AUT. The difference between the received power levels, plus the gain of the standard gain, is the gain of the AUT. This relationship, described in Section 2.6.5, is

$$
G_{A U T}=G_{S G A}+10 \log _{10} \frac{P_{A U T}}{P_{S G A}}
$$

The results of these measurements are included in Table 1 and Table 2, and are compared to the nominal values provided by the manufacturer. The measured results for the Yagi antenna differ from the nominal value by $1.3 \mathrm{~dB}$, or $15.3 \%$. The measured results for the microstrip array differ from the nominal value by $0.9 \mathrm{~dB}$, or $7.8 \%$.

Table 1: ABSOLUTE GAIN MEASUREMENT, YAGI ANTENNA

$\begin{array}{lllll}\mathbf{P}_{\mathbf{t}}[\mathbf{m W}] & \mathbf{G}_{\text {sg }}[\mathbf{d B i}] & \mathbf{P}_{\text {sg }}[\mathbf{d B m}] & \mathbf{P}_{\text {aut }}[\mathbf{d B m}] & \mathbf{G}_{\text {aut }}[\mathbf{d B i}] \\ 128 & 2 & -41.0 & -35.0 & 8.0 \\ 64 & 2 & -44.9 & -38.9 & 8.0 \\ 32 & 2 & -49.0 & -39.9 & 11.1 \\ 16 & 2 & -50.5 & -41.4 & 11.1 \\ 8 & 2 & -53.6 & -44.9 & 10.7 \\ & \text { Nominal } & \mathbf{8 . 5} & \text { Average } & \mathbf{9 . 8}\end{array}$


Table 2: ABSOLUTE GAIN MEASUREMENT, MICROSTRIP ARRAY ANTENNA

$\begin{array}{lllll}\mathbf{P}_{\mathbf{t}}[\mathbf{m W}] & \mathbf{G}_{\mathbf{s g}}[\mathbf{d B i}] & \mathbf{P}_{\mathbf{s g}}[\mathbf{d B m}] & \mathbf{P}_{\text {aut }}[\mathbf{d B m}] & \mathbf{G}_{\text {aut }}[\mathbf{d B i}] \\ 128 & 2 & -43.6 & -37.6 & 7.9 \\ 64 & 2 & -49.1 & -39.1 & 12.0 \\ 32 & 2 & -51.5 & -41.9 & 11.6 \\ 16 & 2 & -53.9 & -44.3 & 11.6 \\ 8 & 2 & -56.3 & -48.3 & 10.0 \\ & \text { Nominal } & \mathbf{1 1 . 5} & \text { Average } & \mathbf{1 0 . 6}\end{array}$

It is not known how the manufacturer determines the gain of these antennas, or what tolerance is expected in the nominal gain. A different method of determining the gain of these antennas is not available, so the nominal values are used as a guide to determine if the measured results are reasonable. The largest deviation of $1.3 \mathrm{~dB}$ is observed when measuring the Yagi antenna, and demonstrates that the system can provide reasonable absolute gain measurements. Additional errors may be introduced by mechanical misalignment of the antennas, imperfect impedance matching, and by operating in a non-anechoic environment. In particular, the Yagi antenna's reflection coefficient $S_{11}$ proved to be very susceptible to perturbations in its near field. Even placing styrofoam in contact with the antenna's microstrip had a significant effect on the return loss. These near field effects may have contributed to the discrepancy in the gain measurement. However, as the manufacturer provides only the nominal gain, more information would be needed to conclusively judge the gain measurements. The quantization error of the system must be less than $\pm 0.5 \mathrm{~dB}$, and is likely even lower due to the use of dithering. For these reasons, it is likely that the goal of $\pm 0.5 \mathrm{~dB}$ error in the 
gain measure can be achieved in an anechoic environment, provided that particular care is taken to attach the antennas to the positioner without influencing the reactive near field of the antenna. 


\section{FUTURE WORK AND CONCLUSION}

This work has demonstrated that antenna radiation patterns and gain measurements can be produced using a system that costs less than $\$ 1500$ to reproduce. Significant savings in cost and development effort are realized by using Wi-Fi routers as the RF instrumentation. The system's performance is improved by applying a correction coefficient to extend the dynamic range of the router power measurement. A form of dithering is also used to reduce quantization errors. Absolute gain measurements are also made by employing the gain transfer method. The two axis positioner is constructed using readily available materials, and delivers positioning accuracy that rivals commercial systems. When combined with a PC operating LabVIEW software, this permits fully automated antenna pattern measurements.

This system was chosen as a finalist in the 2011 IEEE AP-S Symposium Student Design Challenge. The system was presented at the symposium in Spokane, Washington. This required transporting the entire system to Spokane so that it could be demonstrated before judges and symposium participants. The system was successfully disassembled, packed into two large suitcases, and flown to Spokane. At the conference the system was

reassembled and demonstrated successfully. The system placed $3^{\text {rd }}$ in the design challenge, and was awarded a certificate and cash prize.

The performance of the system may be further improved by exploring the use of improved gain measurement techniques and methods to reduce the effect of reflections from the ground. In this work, gain measurements are conducting using the gain transfer method. This entails comparing the performance of an unknown antenna against an SGA 
with known gain. A dipole is used as the SGA in this work. The dipole is not a highly directive antenna, therefore, the effect of reflections from the environment may be more pronounced. The use of a more directive SGA may improve the gain measurement results when gain transfer methods are used.

The system delivered gain measurements that were comparable to the manufacturer specified gain of the test antennas. However, using test antennas whose gain is known with certainty would allow the system's gain measurement ability to be more conclusively evaluated. By using antennas with certified gains, the system's ability to deliver gain measurements within $0.5 \mathrm{~dB}$ could be confirmed.

During the symposium, one attendee suggested a method of reducing the effect of ground reflections. Figure 20 and Figure 21 show that ground reflections can have a significant effect on pattern measurements. When pattern measurements are conducted at the outdoor ranges at the National Institute of Standards and Technology (NIST), the results may be influenced by reflections off the ground. Because the amount of reflection is not precisely known, and may vary based on factors such as soil composition and moisture content, it is difficult to subtract the effect of this reflected energy from the result. To allow the effects of ground reflections to be subtracted from the measured result, the ground is covered with conductive mesh. This causes the surface to act as a continuous PEC surface. The behavior of electromagnetic energy reflected off of this surface can be determined using geometric optics methods. Once determined, this energy can then be subtracted from the power received by the AUT. This greatly reduces the effect of ground reflections on pattern measurements. 


\section{CITED LITERATURE}

1. C. A. Balanis, Antenna Theory: Analysis Design, 3rd ed. Hoboken, NJ: Wiley, 2005, pp. 1001.

2. C. A. Balanis, Antenna Theory: Analysis Design, 3rd ed. Hoboken, NJ: Wiley, 2005, pp. 1021.

3. C. A. Balanis, Antenna Theory: Analysis Design, 3rd ed. Hoboken, NJ: Wiley, 2005, pp. 150.

4. IEEE Standard Test Procedures for Antennas, IEEE Std 149-1979, Published by IEEE, Inc., 1979, p 25.

5. C. A. Balanis, Antenna Theory: Analysis Design, 3rd ed. Hoboken, NJ: Wiley, 2005, pp. 1001.

6. W. L. Stutzman and G. A. Thiele, Antenna Theory and Design. New York: Wiley, 1981, pp. 22.

7. W. L. Stutzman and G. A. Thiele, Antenna Theory and Design. New York: Wiley, 1981, pp. 23.

8. W. H. Kummer and E. D. Gillespie, “Antenna Measurements-1978,” Proc. IEEE., vol. 66, no. 4, pp. 485, Apr. 1978.

9. C. A. Balanis, Antenna Theory: Analysis Design, 3rd ed. Hoboken, NJ: Wiley, 2005, pp. 1004.

10. IEEE Standard Test Procedures for Antennas, IEEE Std 149-1979, Published by IEEE, Inc., 1979, p 25.

11. M. D. Foegelle, “Antenna Pattern Measurement: Concepts and Techniques,” Compliance Engineering Magazine, 2002 Reference Guide.

12. IEEE Standard Test Procedures for Antennas, IEEE Std 149-1979, Published by IEEE, Inc., 1979, p 25.

13. C. A. Balanis, Antenna Theory: Analysis Design, 3rd ed. Hoboken, NJ: Wiley, 2005, pp. 1025.

14. C. A. Balanis, Antenna Theory: Analysis Design, 3rd ed. Hoboken, NJ: Wiley, 2005, pp. 1027. 


\section{CITED LITERATURE (continued)}

15. C. A. Balanis, Antenna Theory: Analysis Design, 3rd ed. Hoboken, NJ: Wiley, 2005, pp. 95.

16. IEEE Standard Test Procedures for Antennas, IEEE Std 149-1979, Published by IEEE, Inc., 1979, p 78.

17. C. A. Balanis, Antenna Theory: Analysis Design, 3rd ed. Hoboken, NJ: Wiley, 2005, pp. 1031.

18. W. H. Kummer and E. D. Gillespie, “Antenna Measurements-1978,” Proc. IEEE., vol. 66, no. 4, pp. 495, Apr. 1978.

19. IEEE Standard for Information Technology. Local and metropolitan area networks Specific requirements. Par 11: Wireless LAN Medium Access Control and Physical Layer Specifications, section 14.2.3.2, IEEE Std 802.11-2007, 2007.

20. “Converting Signal Strength Percentage to dBm Values,” WildPackets, Inc., Walnut Creek, CA, Nov. 2002.

21. R. H. Bishop, LabVIEW 8 Student Edition, 1st ed. Prentice Hall, 2007.

22. R. Schmidt, “Digital Signals - Sampling and Quantization,” RS-MET, Berlin.

23. B. Bannon, “Overcoming Converter Nonlinearities with Dither,” Analog Devices, Norwood, MA, AN-410 Application Note, Dec. 1995.

24. Satimo. Standard Gain Horns [Online]. Available: http://www.satimo.com/content/products/standard-gain-horns

25. K. J. Kaczmarski, “An “exact” inverse source reconstruction,” Ph.D dissertation, Dept. Elect. Eng., Univ. Illinois, Chicago, 2008. 


\section{VITA}

NAME: $\quad$ Keith Robert Martin

EDUCATION: $\quad$ M.S., Electrical and Computer Engineering, University of Illinois at Chicago, Chicago, Illinois, 2011, under the supervision of Prof. Danilo Erricolo

B.S., Electrical Engineering, Northwestern University, Evanston, Illinois, 2007

PROFESSIONAL U.S. Cellular, Itasca, Illinois

EXPERIENCE: $\quad$ Associate RF Engineer, 2011

Accenture, Chicago, Illinois

Systems Integration and Technology Analyst, 2008-2010

PEC Products, Leuven, Belgium

Electrical Engineer, Battery Business Solutions, 2007-2008

MEMBERSHIP: IEEE Eta Kappa Nu Honor Society 